\title{
The mass-ratio and eccentricity distributions of barium and $S$ stars, and red giants in open clusters ${ }^{\star}$
}

\author{
M. Van der Swaelmen ${ }^{1}$, H. M. J. Boffin ${ }^{2,3}$, A. Jorissen ${ }^{1}$, and S. Van Eck ${ }^{1}$ \\ ${ }^{1}$ Institut d'Astronomie et d'Astrophysique, Université Libre de Bruxelles, Campus Plaine C.P. 226, Bd du Triomphe, 1050 Bruxelles, \\ Belgium \\ e-mail: [ajorisse; svaneck] @ulb.ac.be \\ 2 ESO, Alonso de Córdova 3107, Casilla 19001, Santiago, Chile \\ e-mail: hboffin@eso.org \\ 3 ESO, Karl Schwarzschild Strasse 2, 85748 Garching, Germany
}

Received 6 May 2016 / Accepted 11 August 2016

\begin{abstract}
Context. A complete set of orbital parameters for barium stars, including the longest orbits, has recently been obtained thanks to a radial-velocity monitoring with the HERMES spectrograph installed on the Flemish Mercator telescope. Barium stars are supposed to belong to post-mass-transfer systems.

Aims. In order to identify diagnostics distinguishing between pre- and post-mass-transfer systems, the properties of barium stars (more precisely their mass-function distribution and their period-eccentricity $(P-e)$ diagram) are compared to those of binary red giants in open clusters. As a side product, we aim to identify possible post-mass-transfer systems among the cluster giants from the presence of $s$-process overabundances. We investigate the relation between the $s$-process enrichment, the location in the $(P-e)$ diagram, and the cluster metallicity and turn-off mass.

Methods. To invert the mass-function distribution and derive the mass-ratio distribution, we used the method pioneered by Boffin et al. (1992) that relies on a Richardson-Lucy deconvolution algorithm. The derivation of $s$-process abundances in the open-cluster giants was performed through spectral synthesis with MARCS model atmospheres.

Results. A fraction of $22 \%$ of post-mass-transfer systems is found among the cluster binary giants (with companion masses between 0.58 and $0.87 M_{\odot}$, typical for white dwarfs), and these systems occupy a wider area than barium stars in the $(P-e)$ diagram. Barium stars have on average lower eccentricities at a given orbital period. When the sample of binary giant stars in clusters is restricted to the subsample of systems occupying the same locus as the barium stars in the $(P-e)$ diagram, and with a mass function compatible with a WD companion, 33\% (=4/12) show a chemical signature of mass transfer in the form of $s$-process overabundances (from rather moderate - about $0.3 \mathrm{dex}$ - to more extreme - about $1 \mathrm{dex}$ ). The only strong barium star in our sample is found in the cluster with the lowest metallicity in the sample (i.e. star 173 in NGC 2420 , with $[\mathrm{Fe} / \mathrm{H}]=-0.26$ ), whereas the barium stars with mild $s$-process abundance anomalies (from 0.25 to $\sim 0.6 \mathrm{dex}$ ) are found in the clusters with slightly subsolar metallicities. Our finding confirms the classical prediction that the $s$-process nucleosynthesis is more efficient at low metallicities, since the $s$-process overabundance is not clearly correlated with the cluster turn-off (TO) mass; such a correlation would instead hint at the importance of the dilution factor. We also find a mild barium star in NGC 2335 , a cluster with a large TO mass of $4.3 M_{\odot}$, which implies that asymptotic giant branch stars that massive still operate the $s$-process and the third dredge-up.
\end{abstract}

Key words. binaries: spectroscopic - stars: abundances - white dwarfs - open clusters and associations: general

\section{Introduction}

It is difficult to match observational constraints with scenarios of mass transfer involving red giant stars. Most notably the observed distribution of post-mass-transfer systems in the period-eccentricity $(P-e)$ diagram does not match the model predictions because models basically predict abimodal distribution: on the one hand, circular short-period systems $(P<800 \mathrm{~d})$, resulting from Roche-lobe overflow (RLOF), and on the other hand, eccentric systems with periods longer than about $3000 \mathrm{~d}$ that avoided RLOF and always remained detached

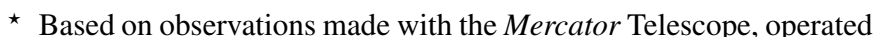
on the island of La Palma by the Flemish Community, at the Spanish Observatorio del Roque de los Muchachos of the Instituto de Astrofisica de Canarias, and on observations made with the HARPS spectrograph installed on the $3.6 \mathrm{~m}$ telescope at the European Southern Observatory.
}

((Boffin \& Jorissen 1988; Pols et al. 2003); Bonačić Marinović et al. 2008; Izzard et al. 2010). Canonical models described in these former studies thus predict a gap with no post-masstransfer systems with periods around $1000 \mathrm{~d}$. However, observations of post-mass-transfer systems like barium stars reveal no such period gap (Jorissen et al. 1998). To make progress on this issue, we perform in this paper a comparative study of the observed properties ( $P-e$ diagram, mass-ratio distribution) of samples of pre-mass-transfer and post-mass-transfer systems.

Barium stars (Bidelman \& Keenan 1951) are a prototypical family of post mass-transfer binaries involving low- or intermediate-mass stars (e.g. McClure et al. 1980; Boffin \& Jorissen 1988; McClure \& Woodsworth 1990; Jorissen et al. 1998). The post-mass-transfer nature of barium stars is made obvious by the chemical anomalies exhibited by the giant primary, for example, strong absorption lines of ionised barium in its 
spectrum and of other elements produced by the $s$ process of nucleosynthesis (Käppeler et al. 2011). The $s$-process material has been transferred to the barium star when the former primary, now a white dwarf (WD), was an asymptotic giant branch (AGB) star. This binary scenario was convincingly confirmed by the observation that (almost) all barium stars reside in binary systems (McClure et al. 1980; McClure 1983; Jorissen \& Mayor 1988; McClure \& Woodsworth 1990; Jorissen et al. 1998). Recently, a radial-velocity monitoring with the HERMES spectrograph (Raskin et al. 2011) attached to the $1.2 \mathrm{~m}$ Mercator telescope from the Katholieke Universiteit Leuven has provided the last remaining orbits (some with orbital periods as long as $50 \mathrm{yr}$ ) for a complete sample of barium stars (Jorissen et al., in prep.).

Here, we make use of this complete set of post-mass-transfer orbital elements to study the resulting mass function distribution to revisit the mass-ratio distribution of these stars, therefore extending the work of McClure \& Woodsworth (1990), Boffin et al. (1992), and Jorissen et al. (1998). For post-masstransfer systems such as barium stars, the companion should be a $\mathrm{CO}$ white dwarf with a mass larger than $0.51 M_{\odot}$ (for a star of initial mass $0.9 M_{\odot}$; e.g. Eq. (66) of Hurley et al. 2000). This prediction may be tested from the mass-ratio distribution of barium stars. But before embarking onto this, it is useful to have a comparison sample. In their pioneering work, Boffin et al. (1993) constructed a sample of normal, field $\mathrm{G}-\mathrm{K}$ giants known to be spectroscopic binaries and found that the mass ratio distribution was most likely close to uniform. This had the advantage of having field stars similar to the sample of barium stars, allowing for a better comparison. On the other hand, this sample was very heterogeneous, coming from different sources. Since it was not possible to know the masses of these stars, some assumptions had to be made. Since then, we are fortunate enough that a new, homogeneous catalogue has been published. Mermilliod et al. (2007) indeed provide spectroscopic orbits for 156 red giants in open clusters, as the final outcome of a very long programme carried out with the CORAVEL instrument (Baranne et al. 1979). We use this sample as a comparison to our sample of barium-star orbits (and their cooler analogues, the extrinsic S stars).

The paper is constructed as follows. In Sect. 2, we present our method to analyse the mass ratio distribution of a sample of binary stars, which we then apply to the sample of Mermilliod et al. (2007) in Sect. 3 and to our enlarged sample of barium and S stars in Sect. 4. Section 5 describes an abundance analysis that aims to detect barium stars in open clusters.

\section{Methodology}

For spectroscopic binary systems with only one observable spectrum (SB1), the individual component masses cannot be accessed directly; they instead combine in the spectroscopic mass function $f(m)$ :

$f(m)=\frac{M_{2}^{3}}{\left(M_{1}+M_{2}\right)^{2}} \sin ^{3} i=M_{1} \frac{q^{3}}{(1+q)^{2}} \sin ^{3} i$,

where $i$ is the (unknown) orbital inclination with respect to the plane of the sky, $M_{1}$ and $M_{2}$ are the primary and secondary masses, respectively, and $q=M_{2} / M_{1}$ is the mass ratio. The mass function (expressed in solar masses $M_{\odot}$ ) is derived from observable quantities

$f(m)=1.038510^{-7} K_{1}^{3}\left(1-e^{2}\right)^{3 / 2} P$,

where $P$ is the orbital period (expressed in days in the above relation), $K_{1}$ (in $\mathrm{km} \mathrm{s}^{-1}$ ) is the radial-velocity semi-amplitude of the observable primary component, and $e$ is the eccentricity.
In our case, the primary is the red giant, so if there is a way to know (or assume) its mass, we can, for a given sample, study the distribution of the quantity $Y=f(m) / M_{1}$. As we can safely assume that the orbital inclination is randomly distributed according to $g(i)=\sin i$, the distribution of $Y$ can thus provide us with the distribution of mass ratios, $d(q)$, that we are looking for (see e.g. Boffin et al. 1992, 1993; Cerf \& Boffin 1994; Pourbaix et al. 2004; Boffin 2010, 2012).

One way to accomplish this is to look at the distribution of $\log Y$ (and not of $Y$; see Boffin 2010) and compare this with some given a priori distributions. Another way is to use a method to numerically invert the equation that links the observed distribution of $Y$ with that of $q$. Here, we use the method designed by Boffin et al. (1992), which relies on a Richardson-Lucy deconvolution and has proven to be very robust and reliable (see references above).

\section{Binary red giants in open clusters}

Mermilliod et al. (2007, M07 in the following) obtained radial velocities of 1309 red giants in 187 open clusters distributed over the whole sky. These red giants have been monitored with the CORAVEL and CfA spectrometers for $20 \mathrm{yr}$, with a typical accuracy of $0.4 \mathrm{~km} \mathrm{~s}^{-1}$ per observation. They detected 289 spectroscopic binaries and published orbits for 156 systems with an average of 26 observations per system. The orbital periods range from 41.5 days to $40 \mathrm{yr}$ and eccentricities from 0 to 0.8 . The remaining 133 systems have periods that are too long, an insufficient number of observations, and/or inadequate phase coverage for an orbit determination.

Although the M07 sample of binary red giants in open clusters cannot be considered complete for the reasons listed above, the homogeneity of the data and the observing strategy nevertheless permit a reliable assessment of the statistical properties of the binary systems of this sample. In particular, one may safely assess that the major incompleteness concerns the systems with the longest orbital periods, above $10^{4} \mathrm{~d}-$ about $30 \mathrm{yr}-$, given that the velocity monitoring spanned $20 \mathrm{yr}$. This selection effect does not bias the intended comparison with the post-masstransfer sample of barium stars, which has a similar period cutoff, as we discuss below (Sect. 4). Another advantage of the M07 sample is that it deals with members of open clusters. We can use the latter to estimate the mass of the red giant needed for Eq. (1). This was carried out in the following way: for each cluster, we used the distance, reddening, age, and metallicity of the clusters as collected in WEBDA ${ }^{1}$. The photometric data of the objects are then used to locate them into a Hertzsprung-Russell diagram. We then used the $\mathrm{BaSTI}^{2}$ isochrones corresponding to the adequate metallicity, and corrected for the given reddening and distance, to determine the mass of the stars. We preferred to use this method than simply use the turn-off of the cluster as the mass of the giants, as this should lead to a more precise value and an independent check of membership of the star. In fact, this methodology was usable on only 124 systems, which is the sample we use in the following. Of the 32 systems for which we could not find an adequate solution, 28 were flagged by M07 as non-cluster members but field stars. The remaining four (NGC 2489 25, NGC 2925 92, Ru 79 2, and Tr 26 201) are supposedly cluster members, but we do not consider them further here, as their small number is not going to degrade the statistics.

\footnotetext{
1 http://www. univie.ac.at/webda/

2 http://albione.oa-teramo.inaf.it/
} 


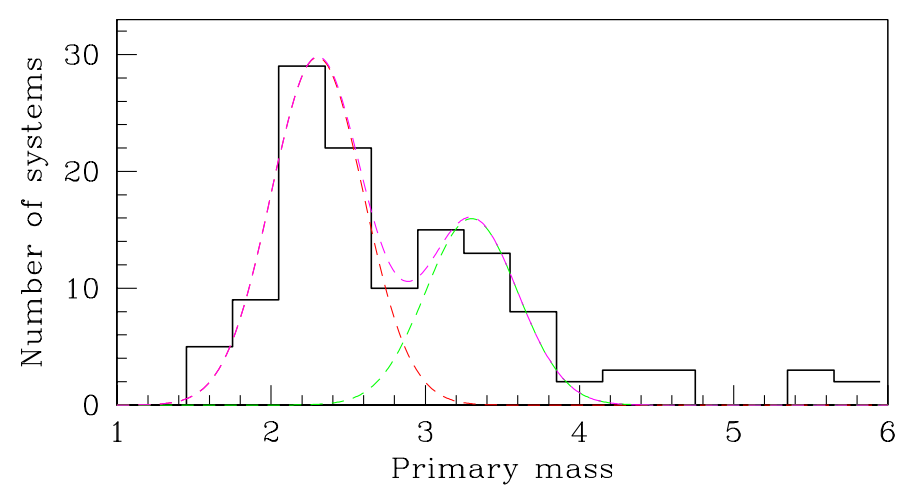

Fig. 1. Distribution of the primary masses, $M_{1}$, for the M07 sample of red giants is shown as the black histogram. We also show a Gaussian distribution centred on $2.3 M_{\odot}$ and with $\sigma=0.3 M_{\odot}$ with the red dashed line, while the thin dashed green line is a Gaussian centred on $3.3 M_{\odot}$ and with $\sigma=0.3 M_{\odot}$.

The resulting mass distribution is shown in Fig. 1 and Table A.4. Typical errors on the mass we so derive are between $0.05 M_{\odot}$ and $0.2 M_{\odot}$, i.e. between $1 \%$ and $9 \%$. Such a small error has no consequence on the mass ratio distributions we derive, given the weak dependence of the mass ratio on the primary mass, for a given spectroscopic mass function (see Eq. (1)). The bulk of the primary-mass distribution we obtain can be approximated by a Gaussian distribution centred on $2.3 M_{\odot}$ and with a standard deviation $\sigma=0.3 M_{\odot}$, although there are some additional systems with masses between 3 and $4 M_{\odot}$ (with a secondary peak at $3.3 M_{\odot}$ ). The mean mass of the red giants in this sample is $2.9 M_{\odot}$. In the following, we carry out the analysis using either the actual primary-mass distribution as determined through isochrone fitting or using a single Gaussian distribution as first approximation.

The resulting distribution of $\log Y$ for the M07 sample, using the primary masses we determined, is shown in Fig. 2, where we also show the distribution (cyan dashed line in the bottom panel) we would expect if we had a uniform mass-ratio distribution (MRD), as was found by Boffin et al. (1993) for their sample of red giant spectroscopic binaries. Clearly, the observed distribution has an over density of systems with $-3<\log Y<-2$ compared to the uniform distribution. As an illustration, a value of $\log Y=-2$ corresponds to a mass ratio of 0.3 , when taking the mean value of $\sin ^{3} i$. For a primary mass of $2.3 M_{\odot}$, such a mass ratio corresponds to a secondary mass of $0.69 M_{\odot}$. Thus, it appears that the observed distribution of $\log Y$ indicates that there are more systems with such a mass ratio (or corresponding secondary mass) than one would expect from a uniform distribution. There is no reason to expect such an excess of 0.6-0.7 $M_{\odot}$ stars, if they were on the main sequence, unless some process during star formation led to a peak at this mass ratio. This is, however, neither seen in the analysis of field red giant stars, nor in the study of solar-like stars (Halbwachs et al. 2003). Such a mass does correspond, however, to the typical mass of CO WDs (Falcon et al. 2010; Kleinman et al. 2013), and one could thus assume that there is a fraction of systems in the M07 sample that are post-mass-transfer systems, i.e. systems in which the (present) red giant was initially the least massive of the two stars and the primary already evolved to the WD stage. Such systems are similar to barium and S stars, although they may not all be contaminated in $s$ process (this is checked in Sect. 5.1), and would be the possible descendants of blue straggler stars seen in open clusters. Such post-mass-transfer giants should thus be somewhat bluer (about $0.05-0.07 \mathrm{mag}$ in $B-V$ for a mass

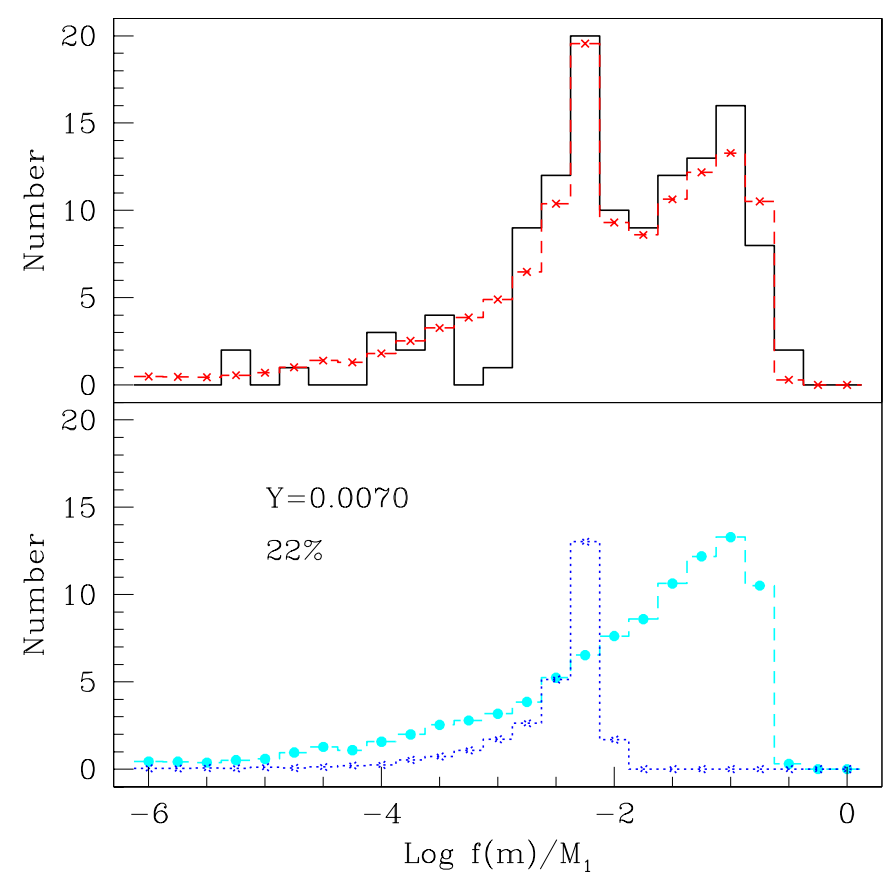

Fig. 2. Upper panel: black histogram shows the distribution of the logarithm of the reduced mass function, $Y=f(m) / M_{1}$, for the sample of spectroscopic binaries from M07, while the red dotted line connecting filled dots is our best model (see text). Lower panel: this shows the two components entering into our best-fit model using a Monte Carlo approach: The cyan dashed line connecting solid dots is the distribution that one would expect for a uniform mass-ratio distribution, while the blue dotted line connecting crosses corresponds to having $22 \%$ of systems with a Gaussian distribution of $Y$, centred around $Y=0.007$ and a standard deviation of 0.0009 . The best-fit red line in the upper panel is the sum of these two distributions.

difference of $0.2 M_{\odot}$ ) than the other giants. This effect was not seen when the giant masses were derived from isochrone fitting (see earlier in this section), which is probably because the available photometric data was not accurate enough.

We have therefore tried to add, on top of the uniform massratio distribution, a sample of post-mass-transfer systems, represented by systems with a peaked Gaussian distribution of $\langle Y\rangle=Y / \sin ^{3} i$, as was found for barium stars (Webbink 1988, see also Sect. 4). Such a peaked distribution would mean that the mass ratio is peaked, as expected for a WD mass distribution. We have therefore searched for the best fit, by means of $\chi^{2}$ minimisation to the observed distribution, in which we add three free parameters: the fraction of systems belonging to the post-mass-transfer population, $n_{\mathrm{p}}$, and the mean and standard deviation of the Gaussian distribution, $Y_{\mathrm{p}}$ and $\sigma_{\mathrm{p}}$. The result of the best fit, corresponding to a reduced $\chi_{r}^{2}=1.035$, is shown in Fig. 2, and is given by $n_{\mathrm{p}}=0.22 \pm 0.02, Y_{\mathrm{p}}=0.0070$, and $\sigma_{\mathrm{p}}=0.0009 \pm 0.0001$. Such a value of $Y_{\mathrm{p}}$ would translate into a peaked distribution of mass ratios around $q=0.216$, which, assuming the mean mass of red giants in the sample, $2.9 M_{\odot}$, indicates a peaked secondary mass around $0.63 M_{\odot}$, exactly as expected for WDs. For primary masses of 2.3 and $3.3 M_{\odot}$, as suggested from Fig. 1, the secondary mass amounts to 0.50 and $0.71 M_{\odot}$, respectively. We conclude that we need to add $\sim 22 \%$ of post-mass-transfer systems containing a WD to reproduce the observed distribution of $\log Y$ for giants in open clusters.

Of course, instead of assuming some functional form for the MRD and although we seem to be obtaining a very good fit to the observations, it may be more appropriate to use an inversion 


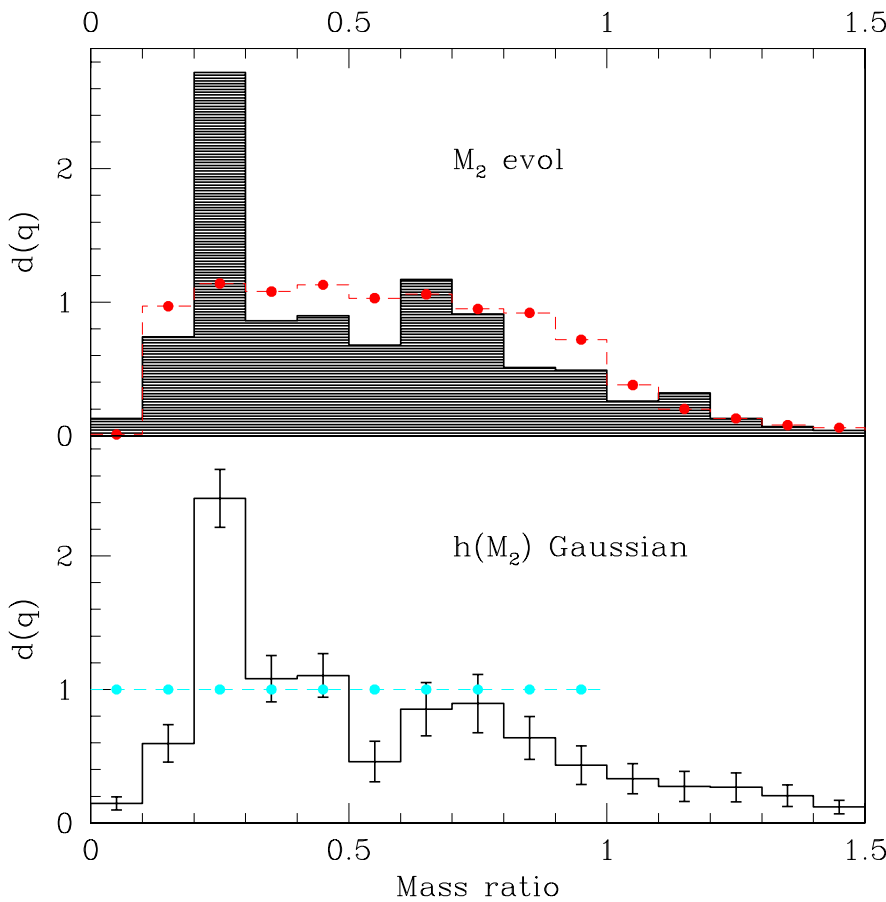

Fig. 3. Mass ratio distribution corresponding to the M07 sample as derived with the Richardson-Lucy algorithm, when using the primary mass derived from the fit of the isochrones (top panel) or assuming a Gaussian distribution (lower panel). For the latter, we have run 1000 simulations and indicate the $1 \sigma$ error bars associated with these In the top panel, we also show, with the red dashed line connecting heavy dots, the distribution we obtain with our inversion method when giving as input a uniform distribution (see text), while in the lower panel the dashed cyan line connecting dots just shows a uniform distribution of mass ratios for comparison.

technique to obtain the MRD directly from the observed distribution of $Y$, without any a priori assumption on the form of the MRD. As mentioned above, this can be carried out with the Richardson-Lucy deconvolution method (Lucy 1974). We have used this method and the results are shown in Fig. 3. In the upper panel, we show the MRD obtained when using the primary masses we derived from isochrones. This figure shows what is now a familiar result: the MRD is generally very uniform (with a small scatter due to the small number of systems) but with an additional strong peak of systems with a mass ratio between 0.2 and 0.3 . For the mean primary mass of this sample, such a mass ratio corresponds to secondary masses between 0.58 and $0.87 M_{\odot}$, i.e. typical masses of WDs, in agreement with what we found above. We also note that our computed distribution shows a few systems with a mass ratio above 1 . As the M07 sample is composed of single-lined binaries with a red giant primary, and since the most massive star should have evolved first, these few systems with $q>1$ are most probably an artefact of the method (due to the limited resolution) and, in some cases, of an underestimate of the primary mass. Similarly, the quasi-absence of systems with mass ratios in the first bin $0<q<0.1$ is most likely due to an observational bias, as systems with very low mass ratios have radial-velocity amplitudes that are too small (and thus too small $f(m)$ ) to be detected. To illustrate this, we show in the top panel of Fig. 3 the result of an experiment we conducted. We created an artificial sample of systems drawn from a uniform distribution of mass ratios, and associated to each of them an inclination, assuming $g(i)=\sin i$. We then added a $10 \%$ error to the so-derived spectroscopic mass function and removed all systems that had a $f(m)$ smaller than the smallest in our sample. We then applied our inversion method to derive the mass ratio distribution and show this as the red dashed curve. We can see that we account for the lack of systems in the first bin and the systems with apparent mass ratio above one.

The lower panel of the same figure shows the MRD we obtain if, instead of using the primary masses we derive from isochrone fitting, we assume that the primary-mass distribution is a Gaussian centred on $2.3 M_{\odot}$ with a standard deviation of $0.3 M_{\odot}$. We have run 1000 simulations, generating the primary mass of a given system according to this distribution every time. This allows us to estimate the typical error bar on each bin in the histogram. As one can see, there is practically no difference resulting from the use of the actual primary-mass distribution or from this Gaussian first approximation. We use this fact later when studying barium and $\mathrm{S}$ stars.

While this work was well underway, we came across the paper by North (2014), which also analysed the sample of cluster giants of Mermilliod et al. as part of a study of A stars. The analysis in this study is, however, restricted to assuming a flat mass ratio distribution for the companions of the red giants, and comparing the distribution of the logarithm of $f(m)$ so obtained, assuming random inclination. North then found, as we did, that the peak in this distribution requires an additional component, which he models as a distribution of WD companions following a Gaussian centred around $0.6 M_{\odot}$ and with a dispersion of $0.03 M_{\odot}$. To reproduce the peak, North (2014) needs a relative number of WD companions of about $23 \%$. His results are thus in agreement with ours, but our method appears more rigorous, with a minimisation method used to find the final set of parameters. Moreover, our inversion technique provides us with a direct mean to obtain the true mass ratio distribution. Finally, North argues that the proportion of $23 \%$ of WD companions can be easily accounted for by assuming a Salpeter initial mass function for the original primaries (the WD progenitors thus) and a uniform (or quasi-uniform) mass-ratio distribution, given the narrow age and mass distributions of the observed giants in the clusters.

\subsection{Looking for the post-mass-transfer objects}

Our results above have shown that the red giant binaries in open clusters contain a fraction, $22 \%$, of systems with a WD companion. As this results from a statistical analysis, it is of course not possible to identify which are these post-mass-transfer systems. One possibility would be to make use of a well-known property of stars in the period-eccentricity $(P-e)$ diagram, as proposed in Jorissen \& Boffin (1992) and Boffin et al. (1993). It is known that most binary samples lack systems with small eccentricities at long periods. Since this gap is already present among premain-sequence binaries (Mathieu 1992), it must be a signature imprinted by the binary formation processes; more precisely, no binary systems form in circular orbits. Subsequent circularisation of the binary system results either from tidal effects (for the shortest systems) or from mass-transfer processes. As a result, the exact extent of this gap, which we call the long-period gap, depends upon the kinds of systems under consideration. In FG main-sequence binaries (Duquennoy \& Mayor 1991) for example, no systems with $e<0.1$ and $P>130$ days are found. But for post-mass-transfer systems such as barium stars, the gap is found at $e<0.05$ and $P>1000 \mathrm{~d}$ (see the top panel of Fig. 4). This narrowing of the gap must clearly be attributed to the mass transfer that occurred in those systems. Therefore, it was hypothesised by Boffin et al. (1993) that the systems with red giants that are in the area $e<0.1$ and $P>130 \mathrm{~d}$ could be post-mass-transfer 
M. Van der Swaelmen et al.: Orbital properties of barium and S stars, and red giants in open clusters

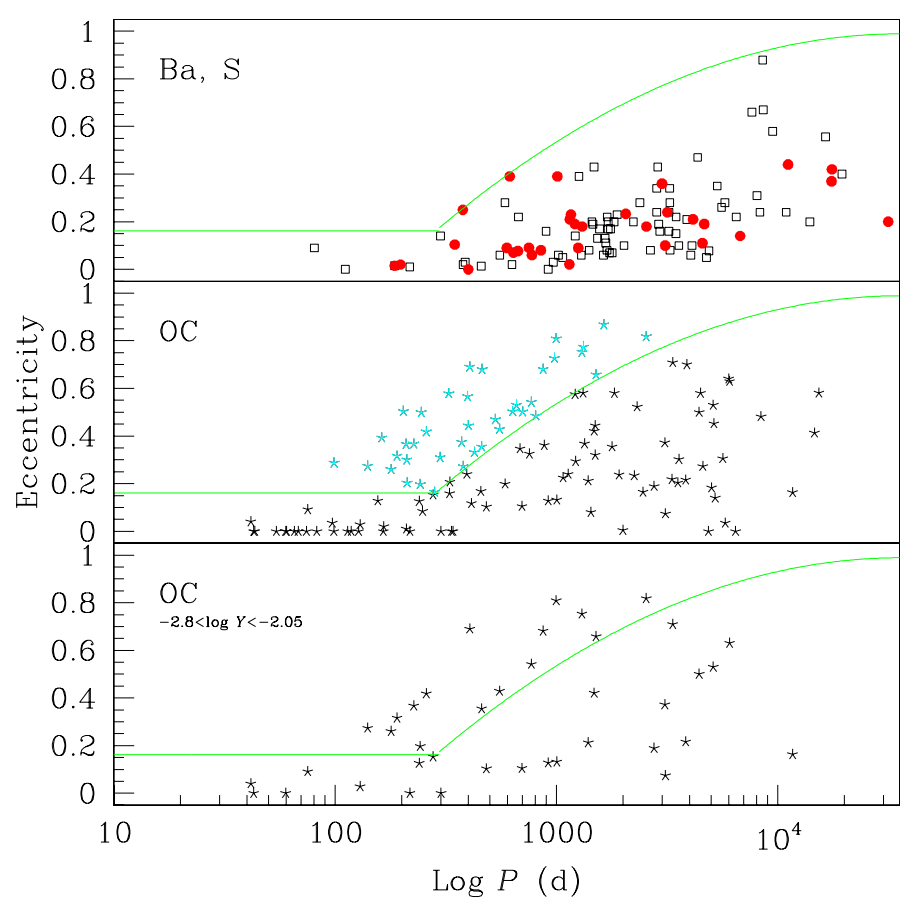

Fig. 4. Orbital period-eccentricity diagrams for the various samples considered here. The upper panel shows orbits of barium (open squares) and $\mathrm{S}$ stars (red dots), as well as a conservative envelope that encompasses all the points (green line). The middle panel shows red giants in binary systems from M07, separated according to the envelope defined above (black and cyan symbols). The lower panel shows only those systems from M07 with mass functions in the range $-2.8<\log Y<-2.05$, that is, those that are more likely to be post-mass-transfer systems (however, about half of these systems are not, according to the bottom panel of Fig. 2).

systems. We test a generalised version of this assumption here using two different methods:

- we look at the $f(m)$ distribution for this subsample and see whether it reveals possibly a larger percentage of WD companions in the sample;

- we perform a chemical abundance analysis for a few stars located in this region with the aim to identify whether they are $s$-process enriched like barium stars.

In addition, we establish the mass-ratio distribution for a subsample of short-period systems and see whether, as expected, the fraction of post-mass-transfer systems has been considerably reduced.

\subsubsection{The mass function}

Figure 4 shows the $(P-e)$ diagram for our sample of barium and $\mathrm{S}$ stars (to be described in Sect. 4) and open cluster stars. As can be seen in the top panel, the eccentricity of barium and S stars is delineated by an envelope, with the mean eccentricity at a given orbital period being smaller for barium and S stars than for normal giants (Boffin et al. 1993) or open cluster giants. To be conservative, we defined this envelope as the curve shown in Fig. 4 and defined by

$$
\begin{aligned}
e=0.16 & \text { if } \log P<2.47 \\
e=-2.90+1.71 \log P-0.19(\log P)^{2} & \text { if } \log P \geq 2.47 .
\end{aligned}
$$

We then separated the sample of M07 giants into two subsamples, depending on which side of the envelope the giants are
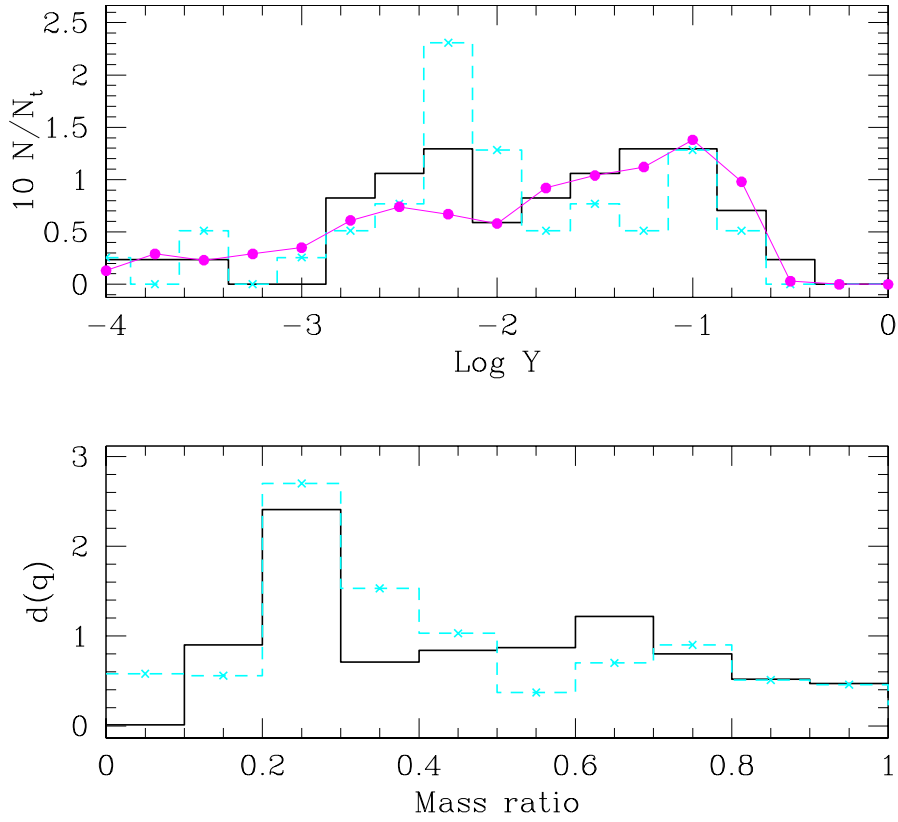

Fig. 5. Distribution of $\log Y$ (top panel) and mass-ratio distribution (bottom) for the two subsamples of M07, according to their position in the $P-e$ diagram; the heavy black curve corresponds to those systems below the envelope shown in Fig. 4, while the cyan dashed line corresponds to those above the envelope. The latter should therefore not contain post-mass-transfer systems, i.e. systems with a WD companion. In the top panel, the magenta line connected by heavy dots represents the distribution expected in case of a uniform distribution. It can be seen that both subsamples deviate from the uniform distribution and the mass ratio distributions are barely distinguishable, even if the systems with large eccentricities seem to be more peaked around $\log Y=-2.3$.

located, with the null hypothesis being that the subsample below the envelope should contain more post-mass-transfer systems, i.e. more systems with a WD companion, while the subsample above the envelope should not contain any. For these two subsamples, we applied the Richardson-Lucy inversion technique to derive the respective mass-ratio distributions as shown in the lower panel of Fig. 5. As can be seen, there is basically no difference between the two subsamples, as both show an excess of low-mass companions.

We also tried the opposite approach. We selected all systems with $-2.8<\log Y<-2.05$ from the M07 sample, i.e. those systems that appear over-represented in Fig. 2 compared to a uniform distribution and which therefore correspond to the white dwarf population. These systems are shown in the $P-e$ diagram in the lower panel of Fig. 4, where it can be seen that they are found on both sides of the post-mass-transfer system's envelope. It should be remembered, however, that the post-mass-transfer systems represent only about half of the systems in the $-2.8<$ $\log Y<-2.05$ range (more precisely $21 / 37=57 \%$ ), as may be judged from Fig. 2.

Thus it appears that it is not at all easy to separate those systems that contain a WD from those that do not in the $P-e$ diagram.

\subsubsection{Chemical enrichment}

We performed an abundance analysis of the giants with long periods and low eccentricities to identify those possibly bearing the chemical signature of mass transfer from a thermally pulsing AGB companion, in the form of overabundances of $s$-process 


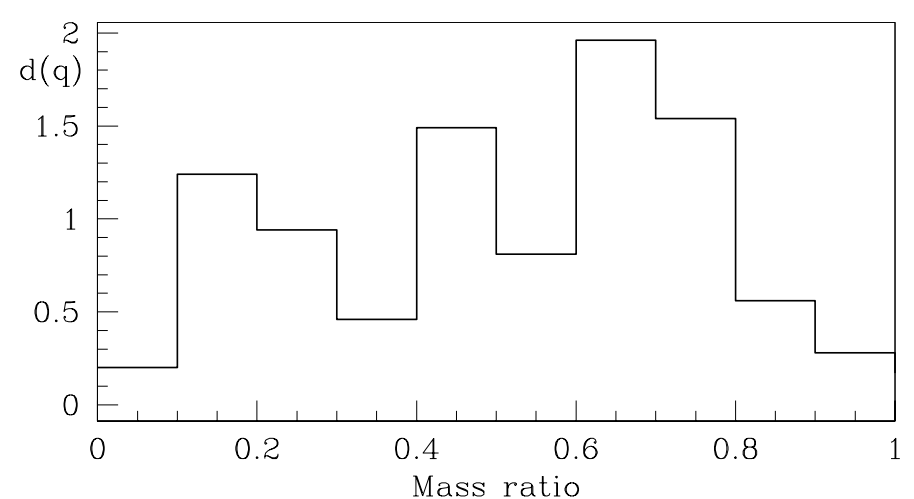

Fig. 6. Mass-ratio distribution corresponding to all the systems from the M07 sample with an orbital period smaller than $180 \mathrm{~d}$, as derived from the Richardson-Lucy algorithm, when using the primary masses derived from the isochrone fits.

elements. In other words, we have looked for possible barium stars among the M07 binaries. Although this abundance study is deferred to Sect. 5.1 below, we present its conclusions here. One barium star with strong anomalies is confirmed in NGC 2420 ( star 173, with $[\mathrm{Fe} / \mathrm{H}]=-0.26$ ), and three more with mild anomalies (of the order of 0.3 to $0.5 \mathrm{dex}$ ) are found in solarmetallicity clusters (Table 3). Among the 12 stars studied [all located in the same region of the $(P-e)$ diagram as barium stars], 4 (or 33\%) thus exhibit some abundance anomalies, a fraction well in line with the expectation from the mass-function analysis.

\subsection{The short-period systems}

Finally, as post-mass-transfer systems should have orbital periods large enough to have avoided the common envelope phase, we should not expect many of these in the M07 sample at small periods. We analysed the mass ratio distribution as obtained with the Richardson-Lucy method for a subsample of M07, taking into account all systems with an orbital period below 180 days, where this value is somewhat arbitrary. In our sample of barium and S stars (see below), we have only two systems with periods below this value. The result is shown in Fig. 6, which, given the small sample (24 systems), is compatible with a uniform distribution of mass ratios, without the need to add any WD companion population.

\section{Barium and S stars}

A companion paper (Jorissen et al., in prep.) complements earlier studies collecting orbital elements for barium and S stars (McClure et al. 1980; McClure 1983; Jorissen \& Mayor 1988; McClure \& Woodsworth 1990; Jorissen et al. 1998), and finally provides the longest orbits among barium and S stars, some with orbital periods up to $50 \mathrm{yr}$. These orbits are obtained in the framework of the ongoing HERMES/Mercator radial-velocity monitoring (Van Winckel et al. 2010; Gorlova et al. 2013); see Jorissen et al. (in prep.) for detailed information. For the sake of completeness, the full list of mass functions, periods, and eccentricities currently available for barium and $\mathrm{S}$ stars is given in Tables A.1-A.3.

In the remainder of this paper (especially Sects. 4.1 and 5.1), it will sometimes be necessary to distinguish between the socalled mild and strong barium stars. This distinction is made on the "Ba index" introduced by Warner (1965), and reflecting the strength of the $\mathrm{Ba}$ lines, based on visual inspection, on a scale

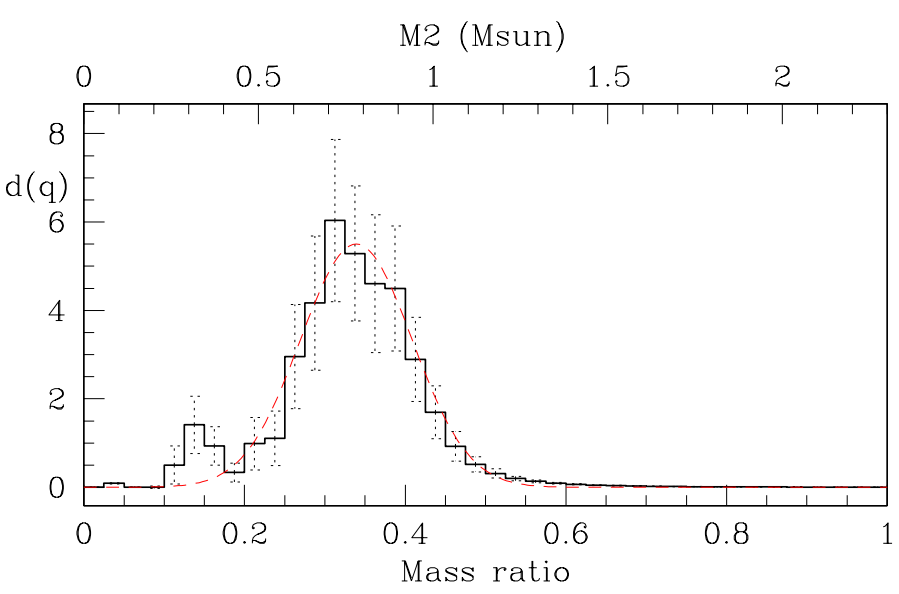

Fig. 7. Mass-ratio distribution for barium stars as derived from the Lucy-Richardson inversion method. A Gaussian fit centred on $q=0.33$ and with $\sigma=0.065$ is shown for comparison (dashed red curve). The upper horizontal scale gives the WD mass, adopting $2.3 M_{\odot}$ for the average mass of the giant star and $0.3 M_{\odot}$ for its standard deviation. The error bars are $1 \sigma$ errors, based on 1000 Monte Carlo simulations.

from $\mathrm{Ba} 1$ to $\mathrm{Ba} 5, \mathrm{Ba} 5$ corresponding to the strongest lines. In this and our past studies, we associate $\mathrm{Ba} 1-\mathrm{Ba} 2$ indices with mild barium stars and $\mathrm{Ba} 3-\mathrm{Ba} 5$ indices with strong barium stars.

\subsection{Mass-ratio distribution}

The derivation of the MRD of barium stars is plagued by the uncertainty existing on their masses. Unlike giants in clusters, there is no direct way to assess the masses of field giants. Mennessier et al. (1997) used a Bayesian method to infer barium-star masses, based on their location in the HertzsprungRussell diagram, using HIPPARCos parallaxes. They conclude that mild and strong barium stars have somewhat different mass distributions, as mild and strong barium stars are characterised by masses in the range $2.5-4.5 M_{\odot}$ and $1-3 M_{\odot}$, respectively. Since the MRD analysis treats mild and strong barium stars together, a distribution that is intermediate between these two seems appropriate. We therefore adopt a Gaussian distribution centred on $2.3 M_{\odot}$ and with a standard deviation of $0.3 M_{\odot}$ for the primary mass of the barium systems, similar to the M07 sample. Fortunately, as shown with our study of the M07 sample, using the real primary-mass distribution or a simplified version of it (Gaussian distribution) does not fundamentally alter the results.

We derived the MRD of barium stars from the 72 orbits listed in Tables A.1, A.2, and adopted the Gaussian mass distribution described above. The outcome of this procedure is shown in Fig. 7, where it is apparent that the distribution is very peaked around $q \sim 0.30$. A Gaussian distribution that best fits the results is centred on $q=0.33$ with a standard deviation of 0.065 . This result is very robust with respect to the choice of the parameters of the barium-star mass distribution $\left(M_{1}\right)$. The average $q$ varies from 0.3 for $\left\langle M_{1}\right\rangle=3 M_{\odot}$ to 0.4 for $\left\langle M_{1}\right\rangle=1.5 M_{\odot}$, whereas $\sigma(q)$ stays at 0.065 . This mass ratio then corresponds to companion masses $M_{2}=0.60,0.76$, and $0.90 M_{\odot}$ for $M_{1}=1.5$, 2.3 , and $3 M_{\odot}$, respectively. The dispersion around these values for the companion mass $M_{2}$ cannot be derived with certainty, since it depends upon the adopted dispersion around $M_{1}$. The value $\sigma(q)=0.065$ derived from the observed mass-function distribution implies $\sigma\left(M_{2}\right)=0.03 M_{\odot}$ if $\sigma\left(M_{1}\right)=0.30 M_{\odot}$ (and $\left.M_{1}=2.3 M_{\odot}\right)$, or $\sigma\left(M_{2}\right)=0.014 M_{\odot}$ if $\sigma\left(M_{1}\right)=0.34 M_{\odot}$. 
In any case, the above masses for the companion are consistent with carbon-oxygen WDs. This is no surprise (McClure \& Woodsworth 1990; Jorissen et al. 1998; North et al. 2000) as barium stars are now well established to be post-masstransfer systems with WD secondaries (McClure \& Woodsworth 1990; Jorissen \& Boffin 1992; Merle et al. 2016). The above result was expressed in a slightly different way by McClure \& Woodsworth (1990) who stated that for barium systems, the value of $Q=M_{2}^{3} /\left(M_{1}+M_{2}\right)^{2}$ can be considered a constant, $Q=$ $0.046 M_{\odot}$, as confirmed in Sect. 4.2. To reconcile the mean value of the WD mass $M_{2}$ in barium systems with that of field DA WDs $\left(0.647 \pm 0.014 M_{\odot}\right.$, from gravitational redshifts; Falcon et al. 2010), a typical barium-star mass of $1.62( \pm 0.20) M_{\odot}$ needs to be adopted. We can only hope to determine precisely the mass of the barium stars with Gaia and its expected delivery of many astrometric binaries. Only then will we be able to check whether or not there is a significant difference in the mass distribution of WDs in barium-star systems and in the field, and thereby provide some constraints on the mass-transfer mechanism.

In Fig. 7, moreover, we see that there is a small excess of systems with mass ratios between 0.1 and 0.2 ; these systems would correspond to lower mass WDs, that is those below $0.46 M_{\odot}$, namely, He WDs. Such WDs cannot have been the core of a thermally pulsing AGB star where the $s$-process material needed to pollute the barium star has been synthesised (Merle et al. 2016). Therefore, this cannot be the correct explanation for the presence of that peak. In fact, the peak may be traced to the presence of the barium star HD 218356, = 56 Peg, with a mass function of $(3.7 \pm 0.3) \times 10^{-5} M_{\odot}($ Table A.2) in the sample. We checked that by removing 56 Peg from our sample, the small peak at $q \sim 0.15$ disappears. A detailed analysis of that system (Frankowski \& Jorissen 2006) concluded that to reconcile constraints from the orbital mass function with evolutionary considerations, the giant must be a fast rotator $\left(V_{\text {rot }} \sim 30-50 \mathrm{~km} \mathrm{~s}^{-1}\right)$ and the orbital inclination must be small $\left(i \sim 5^{\circ}\right)$. This analysis led to masses in the range $2-4 M_{\odot}$ and $0.75-1.15 M_{\odot}$, for the primary and secondary respectively, corresponding to a mass ratio in the range $0.19-0.58$, just above the values $0.1-0.2$ inferred from the statistical analysis of the mass-function distribution. Since HD 218356 is classified as G8 Ib (Morgan et al. 1943), the upper limit of the primary-mass range seems more likely, and yields $q=0.19$. The small inclination of $5^{\circ}$ has a probability of occurrence of one part in 260, and is therefore not expected in a sample of 71 stars such as ours. Therefore, we believe that the secondary peak observed at low $q$ values in Fig. 7 is the result of small-number statistics and does not deserve any further discussion.

We performed the same analysis for our smaller sample of $\mathrm{S}$ star orbits $(N=29)$ and the result is shown in Fig. 8. We obtained a MRD similar to that of barium stars. Both samples are combined in Fig. 9, from which we conclude that S stars are the cooler analogues of barium stars since their orbital properties are similar, in agreement with the conclusion of Jorissen et al. (1998).

\subsection{A different approach, fitting mass functions}

So far, we extracted the MRD from the inversion of the mass-function distribution using the Richardson-Lucy algorithm. Webbink (1988) and McClure \& Woodsworth (1990) have proposed a different approach, fitting the mass-function distribution of barium stars by Gaussian distributions for $M_{1}$ and $M_{2}$ and a random distribution of orbital inclinations. After exploring the parameter space $\left[M_{1}, M_{2}, \sigma\left(M_{1}\right), \sigma\left(M_{2}\right)\right]$, the parameters

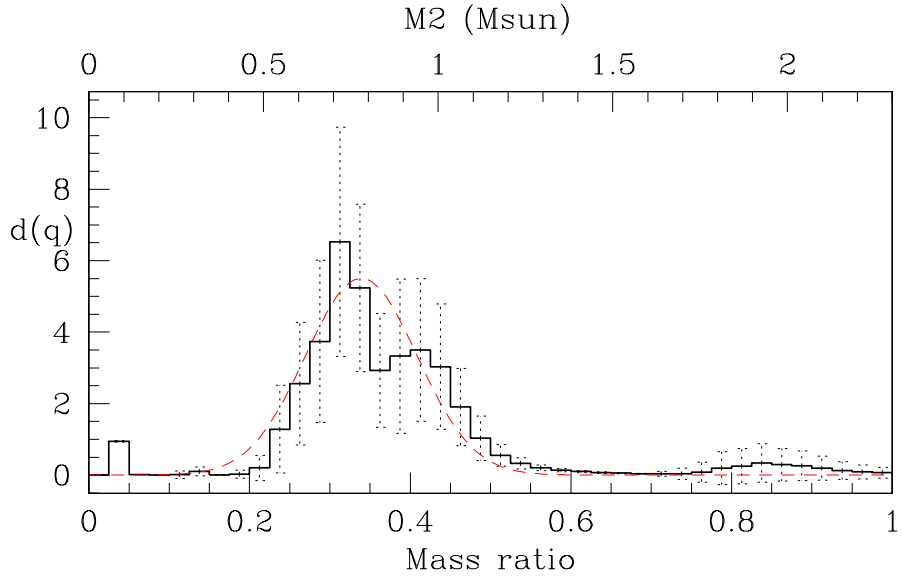

Fig. 8. Same as Fig. 7 for S stars.

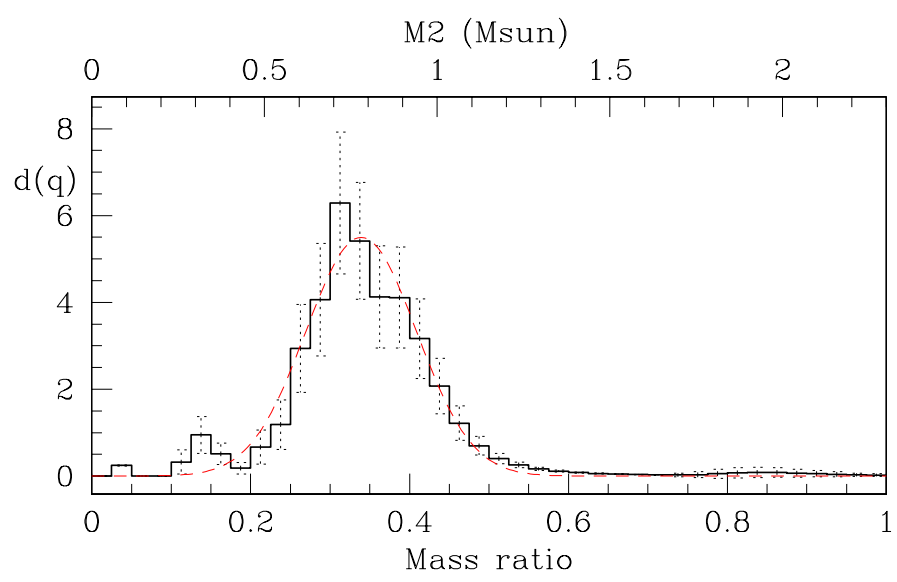

Fig. 9. Same as Fig. 7 for the combined sample of barium and S stars.

minimizing the Kolmogorov-Smirnov distance between the observed and synthetic cumulative functions were considered as yielding the best fit, and were retained. Results from such a best fit of the $f(m)$ distribution are presented in Table 1 and Fig. 10, separately for barium and $\mathrm{S}$ systems.

In Table $1, M_{1}$ and $M_{2}$ denote the central values of the Gaussian distributions with standard deviations $\sigma_{1}$ and $\sigma_{2}$. We stress that the fit actually constrains $Q$, not $M_{1}$ and $M_{2}$ individually. This is why Table 1 contains several $\left(M_{1}, M_{2}\right)$ pairs, which all correspond to $Q=0.041 M_{\odot}$ or $Q=0.045 M_{\odot}$, the values yielding the best fit for barium and $\mathrm{S}$ stars, respectively. These values agree well with the result previously obtained by Jorissen et al. (1998; $\left.Q=0.042 \pm 0.001 M_{\odot}\right)$.

$\mathrm{S}$ stars appear to be lacking small mass functions, and therefore to obtain a good fit, one needs to assume that the systems close to face-on $\left(\cos i>0.85\right.$, or $i<32^{\circ}$ ) are not present in the observed sample. This is not surprising, since small mass functions correspond to systems with small velocity amplitudes, which are difficult to detect if the systems suffer from radialvelocity jitter, as it is the case for evolved giants like $\mathrm{S}$ stars.

The smaller average mass for S-star systems, as compared to barium systems, is expected since (extrinsic) $\mathrm{S}$ stars, which are restricted to low surface temperatures, populate the tip of the RGB; temperature-wise, spectral type $\mathrm{S}$ is equivalent to $\mathrm{M}$, and is thus cooler than K. However, barium stars, with their earlier spectral types (K), could be a mix of RGB stars and He-clump stars because the latter are not restricted to low-mass stars as are RGB stars. 
Table 1. Best-fit parameters for the Gaussian distributions of primary and secondary masses, and $Q \equiv M_{2}^{3} /\left(M_{1}+M_{2}\right)^{2}$, where all masses are expressed in $M_{\odot}$.

\begin{tabular}{lccccc}
\hline \hline Sample & $M_{1}$ & $\sigma_{1}$ & $M_{2}$ & $\sigma_{2}$ & $Q$ \\
\hline $\mathrm{Ba}$ & 1.6 & 0.4 & 0.58 & 0.04 & 0.041 \\
& 1.46 & & 0.55 & & 0.041 \\
& 2.04 & & 0.67 & & 0.041 \\
& 2.46 & & 0.75 & & 0.041 \\
$\mathrm{~S}$ & 1.5 & 0.4 & 0.58 & 0.04 & 0.045 \\
& 1.60 & & 0.60 & & 0.045 \\
\hline
\end{tabular}

Notes. Only $Q$ is constrained by the fit, not $M_{1}$ and $M_{2}$ individually; therefore the first line for each category (Ba or $\mathrm{S}$ ) lists the pairs that were used in the fit, and the following lines list other possible combinations of $M_{1}$ and $M_{2}$ yielding the same $Q$.

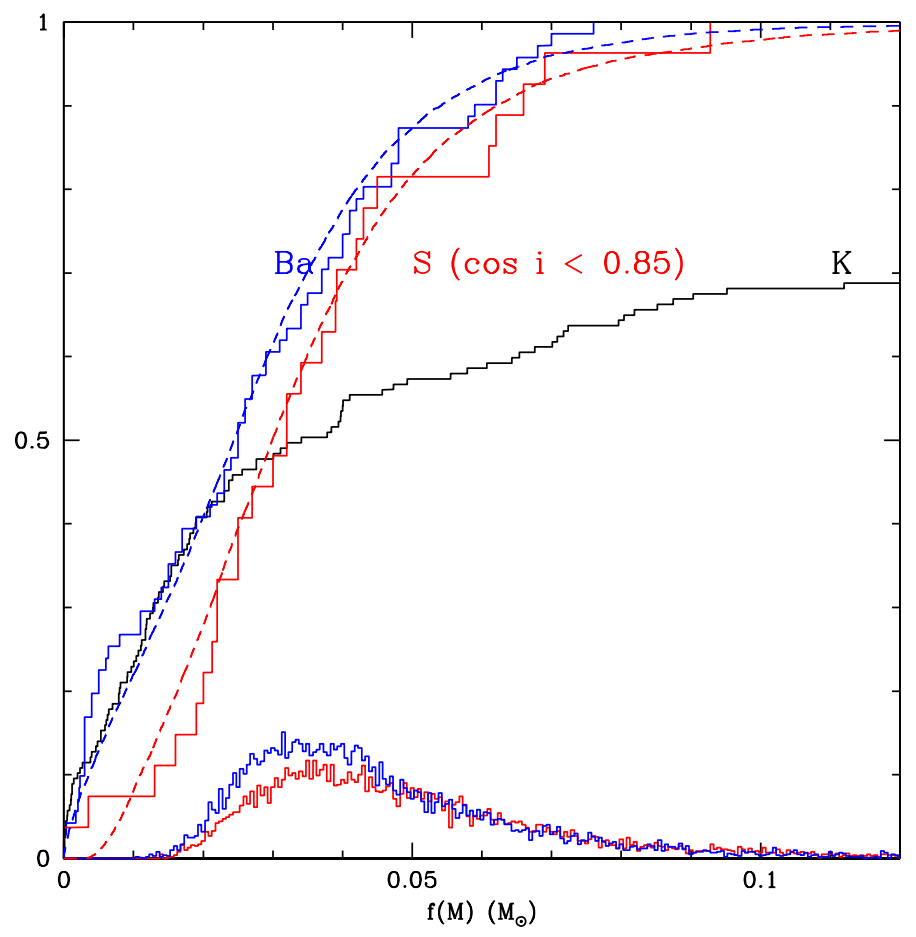

Fig. 10. Cumulative mass-function distributions for barium systems (blue), S-star systems (red), and $\mathrm{K}$ giants in clusters (black). The step curves correspond to the observed values, as listed in Tables A.1-A.3. The dashed curves are the synthetic curves produced by the adopted Gaussian distributions, with parameters as listed on the lines labelled $\mathrm{Ba}$ and $\mathrm{S}$ in Table 1. The histograms at the bottom of the figure correspond to the histograms of $Q$ values for barium and S systems resulting from the normal distributions adopted for $M_{1}$ and $M_{2}$.

A very interesting feature observed in Fig. 10 is the distribution of the mass functions for the binary $\mathrm{K}$ giants in open clusters from M07, which follows exactly that for barium stars, before deviating for frequencies in excess of $43 \%$. This clearly means that at most $43 \%$ of the M07 sample correspond to postmass-transfer systems with WD companions. This frequency is an upper limit, since the low end of the $f(M)$ distribution merges WD and main-sequence companions, whereas the latter distribution becomes dominant for $f(M)>0.02 M_{\odot}$. In any case, this result is consistent with the finding of Sect. 3 based on the inversion of the mass-ratio distribution that $22 \%$ of the M07 binary sample corresponds to post-mass-transfer systems. This prediction makes us suspect that some of the M07 stars should be rich in $s$ process elements as are the barium stars. This prediction is investigated in Sect. 5.

\section{Are there barium stars among the Mermilliod binary giants in open clusters?}

In view of the difficulty in separating pre- from post-masstransfer giants, from simple arguments based on the mass function and the location in the $(P-e)$ diagram (Sect. 3.1), we decided to perform an abundance analysis of those binaries falling in the same region of the $(P-e)$ diagram as barium stars and with a mass function consistent with a WD companion. Such an analysis should uncover barium stars, if there are any hidden in the M07 sample. In the lower panel of Fig. 4, the promising candidates are those located below the green envelope. There are ten such stars that are observable from the Roque de los Muchachos Observatory (Canary Islands, Spain), where the HERMES spectrograph (Raskin et al. 2011) is mounted on the $1.2 \mathrm{~m}$ Mercator telescope from the Katholieke Universiteit Leuven. Two more were observed with the HARPS spectrograph on ESO $3.6 \mathrm{~m}$ telescope. The target properties are listed in Table 2.

On top of these 12 targets, 3 more were analysed because they fall in the long-period gap of the $(P-e)$ diagram (see Sect. 3.1 for a description of this gap). The orbits were recomputed from the available radial-velocity data (as available on the WEBDA database), and with these recomputed eccentricities, one of the binaries (IC 4756 69) is found to fall at the border of the $(P-e)$ gap, but NGC 2682224 and IC 475680 at least remain well within the gap (Table 2 ). On the other hand, only IC 475669 has a $\log Y$ value that makes it compatible with a post-mass-transfer object. These three systems were added at the end of Table 2 as abundance targets as well.

Table 2 also lists four barium stars in open clusters reported in the literature: NGC 2420 250, NGC 2420 173, NGC 5822 2, and NGC 5822 201. The location in the $(P-e)$ diagram of all these cluster stars whose $s$-process abundances were derived are shown in Fig. 11.

Finally, NGC 752208 is observed to serve as a reference star, with no dynamical indication that it could be a post-masstransfer object.

\subsection{Abundances}

For the sake of clarity, all details about the abundance analysis (e.g. derivation of stellar parameters and line lists) are provided in Appendix B. Here we provide only the final abundances (Table 3). The quoted errors on the abundances in that Table are only standard deviations around the mean from line-to-line scatter when several lines are available for a given chemical species. They do not include systematic errors from uncertainties on the model parameters (see Table B.3 for the latter).

The abundances in Table 3 reveal that none of the three stars analysed in the long-period gap in the $(P-e)$ diagram are barium stars nor is the comparison star NGC 752 208. Among the 12 targets with post-mass-transfer properties: one is a strong barium star (NGC 2420 173; see Sect. 4 for the definition of mild and strong barium stars), which is a fact already noted by Mermilliod \& Mayor (2007), but without any quantitative analysis; and three show mild overabundances for at least two $s$-process elements among the five studied (Y, Zr, La, Ce, Nd). The threshold for flagging a star as a mild barium star may be 
M. Van der Swaelmen et al.: Orbital properties of barium and S stars, and red giants in open clusters

Table 2. Binary giants from the M07 sample that were the targets of an abundance analysis.

\begin{tabular}{|c|c|c|c|c|c|c|c|c|c|c|}
\hline Name & $\begin{array}{r}P \\
\text { (d) }\end{array}$ & $e$ & $\log Y$ & $\begin{array}{l}T_{\text {eff }} \\
(\mathrm{K})\end{array}$ & $\log g$ & {$[\mathrm{Fe} / \mathrm{H}]$} & $\begin{array}{l}\text { TO } \\
\left(M_{\odot}\right)\end{array}$ & $\mathrm{Ba}$ & Spectro & Ref. \\
\hline
\end{tabular}

Candidate post-mass-transfer binaries

$\begin{array}{lrlllllllll}\text { NGC 2539 209 } & 11655 & 0.16 & -2.29 & 4750 & 2.5 & +0.13 & 2.6 & \text { N } & \text { HE } & 1 \\ \text { NGC 2335 4 } & 301 & 0.00 & -2.46 & 4750 & 1.2 & -0.03 & 4.3 & \text { Y } & \text { HE } & 1 \\ \text { IC 4756 139 } & 3834 & 0.22 & -2.61 & 5220 & 2.7 & -0.06 & 2.7 & \text { Y } & \text { HE } & 1 \\ \text { NGC 2682 244 } & 698 & 0.11 & -2.22 & 5150 & 2.6 & +0.00 & 1.4 & \text { N } & \text { HE } & 1 \\ \text { NGC 2682 170 } & 4410 & 0.50 & -2.42 & 4250 & 1.7 & +0.00 & 1.4 & \text { N } & \text { HE } & 1 \\ \text { NGC 2682 143 } & 43 & 0.00 & -2.75 & 5000 & 2.8 & +0.00 & 1.4 & \text { N } & \text { HE } & 1 \\ \text { NGC 2420 173 } & 1479 & 0.43 & -2.42 & 5150 & 2.2 & -0.26 & 2.1 & \text { Y } & \text { HE } & 1,2 \\ \text { NGC 6940 111 } & 3571 & 0.30 & -2.05 & 5150 & 2.9 & +0.01 & 2.2 & \text { N } & \text { HE } & 1 \\ \text { NGC 2099 149 } & 918 & 0.13 & -2.48 & 4900 & 2.1 & +0.08 & 3.0 & \text { N } & \text { HE } & 1 \\ \text { NGC 2099 966 } & 3084 & 0.37 & -2.44 & 4600 & 2.1 & +0.08 & 3.0 & \text { N? } & \text { HE } & 1 \\ \text { NGC 2477 1044 } & 3108 & 0.07 & -2.33 & 4780 & 2.6 & +0.01 & 2.1 & \text { Y } & \text { HA } & 1 \\ \text { NGC 4349 203 } & 129.4 & 0.028 & -2.37 & 4940 & 2.2 & -0.07 & 3.2 & \text { N } & \text { HA } & 1\end{array}$

Binaries in the $(P-e)$ gap

$\begin{array}{lllllllllll}\text { IC 4756 69 } & 1994 & 0.05 \pm 0.02 & -2.07 & 4870 & 2.6 & -0.06 & 2.7 & \mathrm{~N} & \text { HE } & 1 \\ \text { IC 4756 80 } & 5791 & 0.03 \pm 0.01 & -1.11 & 4910 & 2.8 & -0.06 & 2.7 & \mathrm{~N} & \text { HE } & 1 \\ \text { NGC 2682 224 } & 6645 & 0.01 \pm 0.01 & -1.04 & 4745 & 2.5 & 0.00 & 1.4 & \mathrm{~N} & \text { HE } & 1\end{array}$

Search for barium stars in clusters, from the literature

\begin{tabular}{|c|c|c|c|c|c|c|c|c|c|c|}
\hline NGC 2420250 & 1404 & 0.08 & -1.64 & - & - & -0.26 & 2.1 & $\mathrm{Y}$ & & 2 \\
\hline NGC 58222 & 1002 & 0.13 & -2.43 & 5100 & 2.4 & -0.15 & 2.1 & Y & & 3 \\
\hline NGC 5822201 & $?$ & $?$ & $?$ & 5200 & 2.7 & -0.15 & 2.1 & $\mathrm{Y}$ & & 3 \\
\hline NGC 5822151 & 1392 & 0.21 & -2.32 & 4900 & 2.5 & -0.11 & 2.1 & $\mathrm{~N}$ & & 4 \\
\hline \multicolumn{11}{|l|}{ Reference star } \\
\hline NGC 752208 & 5214 & $0.13 \pm 0.01$ & -1.35 & 4760 & 2.5 & -0.08 & 1.7 & $\mathrm{~N}$ & $\mathrm{HE}$ & 1 \\
\hline
\end{tabular}

Notes. As before, $Y \equiv f(M) / M_{1}$. The effective temperature $T_{\text {eff }}$ and gravity $\log g$ are derived as explained in Sect. B.1 or taken from the literature. The columns labelled TO and Ba provide the cluster turn-off mass (in $M_{\odot}$ ) and the conclusion of the abundance analysis, respectively: Y (barium star) or N (not a barium star). In column "spectro", HE stands for HERMES and HA for HARPS.

References. (1) This work; (2) Mermilliod \& Mayor (2007); (3) Katime Santrich et al. (2013); (4) Sales Silva et al. (2014).

best evaluated using as reference the abundance dispersion of the sample of field red giants studied by Luck \& Heiter (2007) and Mishenina et al. (2007), which are shown as small crosses in Figs. 12-15 (and non-shaded histogram). These four figures compare the distributions of the $[\mathrm{Y} / \mathrm{Fe}],[\mathrm{La} / \mathrm{Fe}],[\mathrm{Ce} / \mathrm{Fe}]$, and $[\mathrm{Nd} / \mathrm{Fe}]$ abundance ratios in field giants and in the cluster giants targeted for the abundance analysis. From the abundance distribution in field stars, we may assess that $[\mathrm{X} / \mathrm{Fe}]>0.25 \mathrm{dex}$ (where $\mathrm{X}$ stands for any of $\mathrm{Y}, \mathrm{La}, \mathrm{Ce}$, or $\mathrm{Nd}$ ) represents a reasonable threshold to flag a star as a barium star, since very few field giants venture into that region. Based on this criterion, the following three stars are outliers with respect to the field giants for at least two elements (mentioned between parentheses in the following list), and may thus be considered as mild barium stars: IC 4756139 (Zr, La, Ce, Nd), NGC 23354 (Y, Nd), and NGC 24771044 (Y, Zr, La, Nd), and eight are not enriched $([\mathrm{X} / \mathrm{Fe}]<0.25 \mathrm{dex})$ in $s$-process elements (NGC 2099149 , NGC 2099 966, NGC 2539 209, NGC 2682 143, NGC 2682 170, NGC 2682 244, NGC 4349 203, and NGC 6940 111). Thus, among the cluster giants with a mass function that is compatible with a WD companion, $33 \%(=4 / 12)$ show a chemical signature of mass transfer in the form of $s$-process overabundances. None of the three stars in the long-period gap turn out to be a barium star.

Moreover, as shown by Figs. 12-15, the degree of overabundance seems correlated with the cluster metallicity: the 
Table 3. Final $[\mathrm{X} / \mathrm{Fe}]$ abundance ratios for $\mathrm{Y}, \mathrm{Zr}, \mathrm{La}, \mathrm{Ce}$, and $\mathrm{Nd}$ for open cluster stars, normalised by the stellar metallicity.

\begin{tabular}{|c|c|c|c|c|c|c|c|c|c|c|c|}
\hline & {$[\mathrm{Fe} / \mathrm{H}]$} & [Y II/Fe] & & {$[\mathrm{Zr} \mathrm{I} / \mathrm{Fe}]$} & {$[\mathrm{Zr} \mathrm{II} / \mathrm{Fe}]$} & & {$[\mathrm{LaII} / \mathrm{Fe}]$} & {$[\mathrm{CeII} / \mathrm{Fe}]$} & & {$[\mathrm{NdII} / \mathrm{Fe}]$} & $\mathrm{Ba}$ \\
\hline Sun & 0.0 & $-0.01 \pm 0.02$ & 10 & $+0.03 \pm 0.06$ & $3-0.01$ & 1 & $+0.04 \pm 0.07$ & $9-0.03 \pm 0.10$ & 5 & -0 & - \\
\hline IC 4756139 & -0.06 & $+0.02 \pm 0.11$ & 9 & $+0.34 \pm 0.07$ & $3-$ & 0 & $+0.35 \pm 0.10$ & $12+0.18 \pm 0.14$ & 8 & +0.361 & M \\
\hline NGC 2099149 & +0.08 & $+0.00 \pm 0.10$ & 9 & $-0.03 \pm 0.04$ & $2-0.18$ & 1 & $+0.08 \pm 0.14$ & $10+0.08 \pm 0.18$ & 7 & +0.061 & $\mathrm{~N}$ \\
\hline NGC 2099966 & +0.08 & $-0.02 \pm 0.20$ & 9 & $-0.20 \pm 0.03$ & $3-$ & 0 & $+0.20 \pm 0.16$ & $12+0.01 \pm 0.19$ & 6 & +0.081 & $\mathrm{~N}$ \\
\hline NGC 23354 & -0.03 & $+0.46 \pm 0.53$ & 10 & - & $0-$ & 0 & $+0.17 \pm 0.08$ & $6+0.15 \pm 0.22$ & 5 & +0.631 & M \\
\hline NGC 2420173 & -0.26 & $+1.00 \pm 0.28$ & 10 & $+0.72 \pm 0.00$ & $3-$ & 0 & $+0.62 \pm 0.15$ & $7+0.59 \pm 0.12$ & 3 & +1.511 & $\mathrm{~S}$ \\
\hline NGC 2539209 & +0.13 & $-0.02 \pm 0.15$ & 9 & $-0.32 \pm 0.02$ & $2-0.10$ & 1 & $+0.07 \pm 0.12$ & $9+0.02 \pm 0.18$ & 6 & $-\quad 0$ & $\mathrm{~N}$ \\
\hline NGC 2682143 & +0.00 & $+0.06 \pm 0.25$ & 6 & $+0.17 \pm 0.09$ & $3-$ & 0 & $+0.23 \pm 0.14$ & $4+0.04 \pm 0.22$ & 5 & +0.221 & $\mathrm{~N}$ \\
\hline NGC 2682170 & +0.00 & $-0.11 \pm 0.25$ & 6 & $-0.26 \pm 0.11$ & $4-0.23$ & 1 & $-0.10 \pm 0.07$ & $10-0.29 \pm 0.14$ & 4 & $-\quad 0$ & $\mathrm{~N}$ \\
\hline NGC 2682244 & +0.00 & $-0.12 \pm 0.18$ & 11 & $+0.17 \pm 0.09$ & $4-0.28$ & 1 & $-0.08 \pm 0.08$ & $10-0.06 \pm 0.25$ & 6 & +0.061 & $\mathrm{~N}$ \\
\hline NGC 6940111 & +0.01 & $+0.03 \pm 0.16$ & 10 & $+0.39 \pm 0.07$ & $3-0.10$ & 1 & $+0.18 \pm 0.13$ & $13+0.10 \pm 0.22$ & 7 & +0.211 & $\mathrm{~N}$ \\
\hline IC 475669 & -0.06 & $+0.09 \pm 0.10$ & 7 & $-0.02 \pm 0.47$ & $4+0.27 \pm 0.11$ & & $+0.12 \pm 0.08$ & $12-0.03 \pm 0.14$ & 9 & +0.081 & $\mathrm{~N}$ \\
\hline IC 475680 & -0.06 & $+0.03 \pm 0.15$ & 6 & $-0.02 \pm 0.48$ & $4+0.14 \pm 0.09$ & & $+0.07 \pm 0.12$ & $11-0.06 \pm 0.18$ & 9 & +0.101 & $\mathrm{~N}$ \\
\hline NGC 24771044 & +0.01 & $+0.54 \pm 0.10$ & 9 & $+0.29 \pm 0.31$ & $9+0.65 \pm 0.14$ & 3 & $+0.36 \pm 0.13$ & $14+0.16 \pm 0.15$ & 9 & +0.261 & M \\
\hline NGC 2682224 & 0.00 & $+0.03 \pm 0.11$ & 7 & $+0.20 \pm 0.36$ & $8+0.06 \pm 0.15$ & & $+0.09 \pm 0.12$ & $13-0.12 \pm 0.16$ & 10 & +0.141 & $\mathrm{~N}$ \\
\hline NGC 4349203 & -0.07 & $+0.12 \pm 0.07$ & 9 & $+0.03 \pm 0.48$ & $5+0.26 \pm 0.16$ & & $+0.11 \pm 0.13$ & $14-0.05 \pm 0.14$ & 8 & +0.051 & $\mathrm{~N}$ \\
\hline NGC 752208 & -0.08 & $+0.01 \pm 0.15$ & 8 & $+0.22 \pm 0.40$ & $8+0.14 \pm 0.12$ & & $+0.15 \pm 0.10$ & $15-0.04 \pm 0.15$ & 10 & +0.081 & $\mathrm{~N}$ \\
\hline
\end{tabular}

Notes. The quoted errors on the abundances are only standard deviations around the mean from line-to-line scatter when several lines are available for a given chemical species. They do not include systematic errors from uncertainties on the model parameters (see Table B. 3 for the latter). The integer number following the abundances corresponds the number of lines used to compute the mean. The last column flags the star according to the following label: $\mathrm{N}$ (not a barium star), $\mathrm{M}$ (mild barium star), and $\mathrm{S}$ (strong barium star).

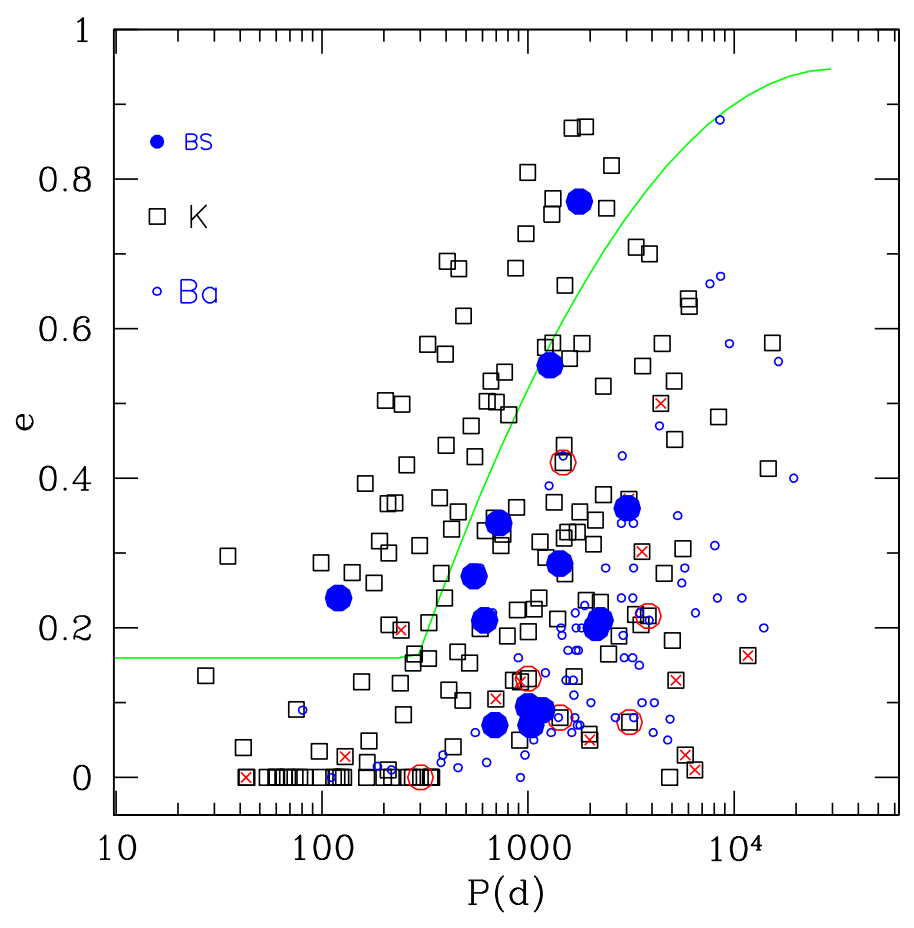

Fig. 11. Period-eccentricity diagram for the M07 sample of binary giants in open clusters (open black squares), barium stars (small open blue circles), and blue-straggler binaries from the cluster NGC 188 (large filled blue dots; Gosnell et al. 2015). M07 cluster giant stars whose $s$-process abundances were derived are identified by red symbols: stars with normal $s$-process abundances are shown as crossed squares, and stars with enhanced $s$-process abundances are shown as circled squares, to be compared with the location of barium stars. The green solid line is the same envelope as that shown in Fig. 4 (see also Eq. (3)).

star NGC 2420 173, which belongs to the most metal-poor cluster of the sample $([\mathrm{Fe} / \mathrm{H}]=-0.26 \mathrm{dex})$, exhibits strong $s$ process overabundances, for elements from the first and second $s$-process peaks. Incidentally, NGC 2420 contains another barium star, NGC 2420250 (Mermilliod \& Mayor 2007). Two among the mild barium stars are found in the clusters with a slightly subsolar metallicity, IC 4756 and NGC 2335 (with $-0.10 \leq[\mathrm{Fe} / \mathrm{H}]<0.0)$, as is the case for the barium stars found in NGC $5822([\mathrm{Fe} / \mathrm{H}]=-0.15 \mathrm{dex})$ by Katime Santrich et al. (2013) and Sales Silva et al. (2014), whereas the fraction of mild barium stars is very low among solar-metallicity clusters (only one is found in NGC 2477 , with $[\mathrm{Fe} / \mathrm{H}]=0.01 \mathrm{dex}$ ). It is tempting to attribute this correlation to the sensitivity of the $s$-process efficiency with metallicity, as predicted, for example, by Goriely \& Mowlavi (2000). Another explanation, in terms of the dilution factor of the accreted matter in the giant's envelope, is not supported by our results (Fig. 16). This is because in the cluster with the lowest turn-off (TO) mass, NGC 2682, none of the three stars studied turn out to be a barium star (even though NGC 2682143 , with $[\mathrm{La} / \mathrm{Fe}]=0.23$ dex, is a close call). Therefore, the dilution factor is not the main factor controlling the level of $s$-process abundances in this cluster where giants have a small envelope mass and, thus, it should be easy to form barium stars. Overall, there is no correlation between $[\mathrm{La} / \mathrm{Fe}]$ and the TO mass, as shown in Fig. 16.

A last comment regarding the TO mass concerns the cluster NGC 2335, which has a large TO mass of $4.3 M_{\odot}$. It is remarkable that it hosts a mild barium star, as diagnosed from its $[\mathrm{Y} / \mathrm{Fe}]$ and $[\mathrm{Nd} / \mathrm{Fe}]$ abundances $(+0.46$ and +0.63 dex, respectively); this means that rather massive AGB stars are still able to operate the $s$ process.

\subsection{Discussion}

The discovery of barium or S stars in open clusters is a real golden nugget as they represent a very definite signature of postmass-transfer objects. Katime Santrich et al. (2013) report the existence of two barium stars in the open cluster NGC 5822, one of which is among the sample of M07: NGC 58222 is known to have an orbital period of 1002.2 days and an eccentricity of 


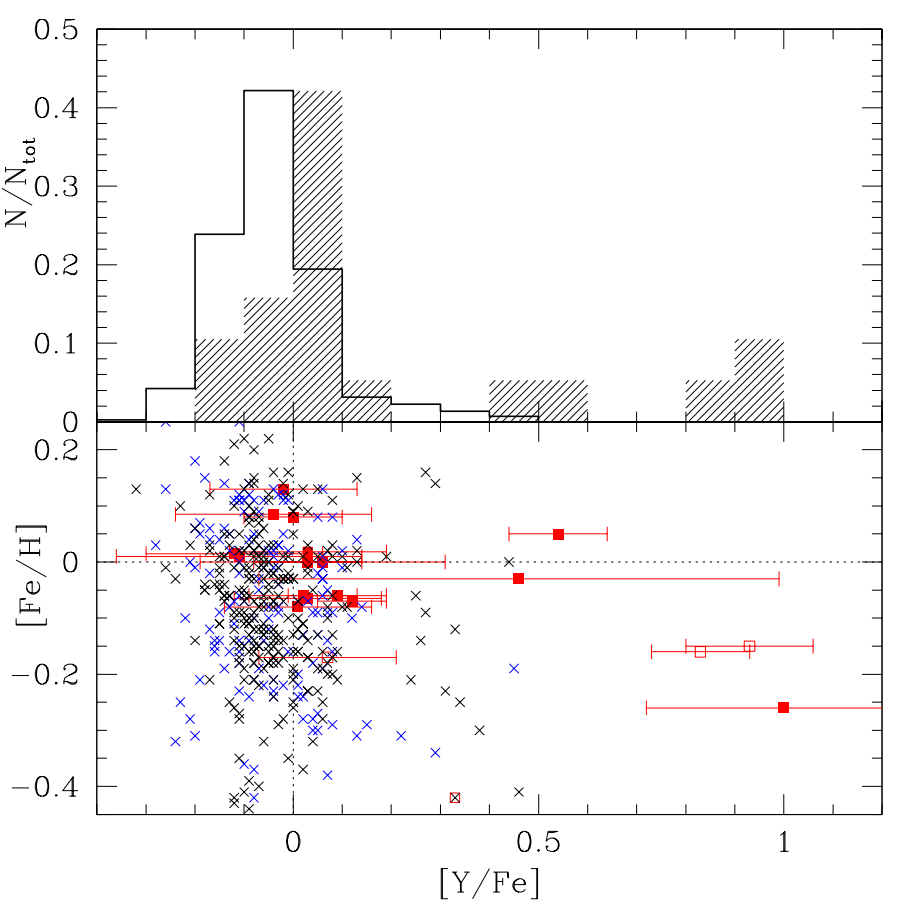

Fig. 12. Bottom panel: relation between $[\mathrm{Y} / \mathrm{Fe}]$ and metallicity $[\mathrm{Fe} / \mathrm{H}]$ for cluster giants analysed in the present study (red filled squares). Literature data (Stars 2, 151 and 201 in NGC 5822, from Katime Santrich et al. 2013; Sales Silva et al. 2014) are depicted by red open squares. For the sake of clarity, stars with identical metallicities have been slightly shifted vertically. Field giants, from Luck \& Heiter (2007) and Mishenina et al. (2007), are depicted by small (respectively black and blue) crosses. The barium star HD 104979 is part of the Luck \& Heiter (2007) sample and is identified by a crossed square. Top panel: the normalised distribution of $[\mathrm{Y} / \mathrm{Fe}]$ for the field giants is represented by the unshaded histogram. The shaded histogram corresponds to the cluster giants (including some barium stars) from the present study.

0.132. As such, it does follow the trend shown by barium and $\mathrm{S}$ stars to have smaller eccentricities than normal giants at a given orbital period. However, in the same cluster, the M07 sample contains two other spectroscopic binaries: NGC 5822151 and NGC 5822 276. Sales Silva et al. (2014) showed that the former is not a barium star. This binary has a period of $1391.5 \mathrm{~d}$ and an eccentricity of 0.212 . It does therefore fall within the (field) barium star locus in the $(P-e)$ diagram, despite not being a barium star, thus confirming the conclusion already apparent from Fig. 11 that M07 cluster giants contain a mix of barium and nonbarium stars at long periods and small eccentricities.

The barium star NGC 58222 has a spectroscopic mass function of $f(m)=0.00916 M_{\odot}$ and an estimated mass of $2.49 M_{\odot}$, i.e. $\log Y=-2.43$, putting it right in the middle of the systems containing a WD. This is thus no surprise. However, this is also the case of NGC $5822151(\log Y=-2.15)$, which is not a barium star.

Our abundance study described in Sect. 5.1, involving 12 more giants from the M07 sample, has generalised the above conclusion that the locus of barium stars in the $(P-e)$ diagram also contains non-barium stars. It is unfortunately not possible to infer the nature of the companion (WD or low-mass mainsequence star) so as to decide whether the non-barium nature of these stars is a consequence of their pre-mass-transfer status or a consequence of mass transfer of matter that is not enriched in $s$ process. The statistics of barium stars found in clusters

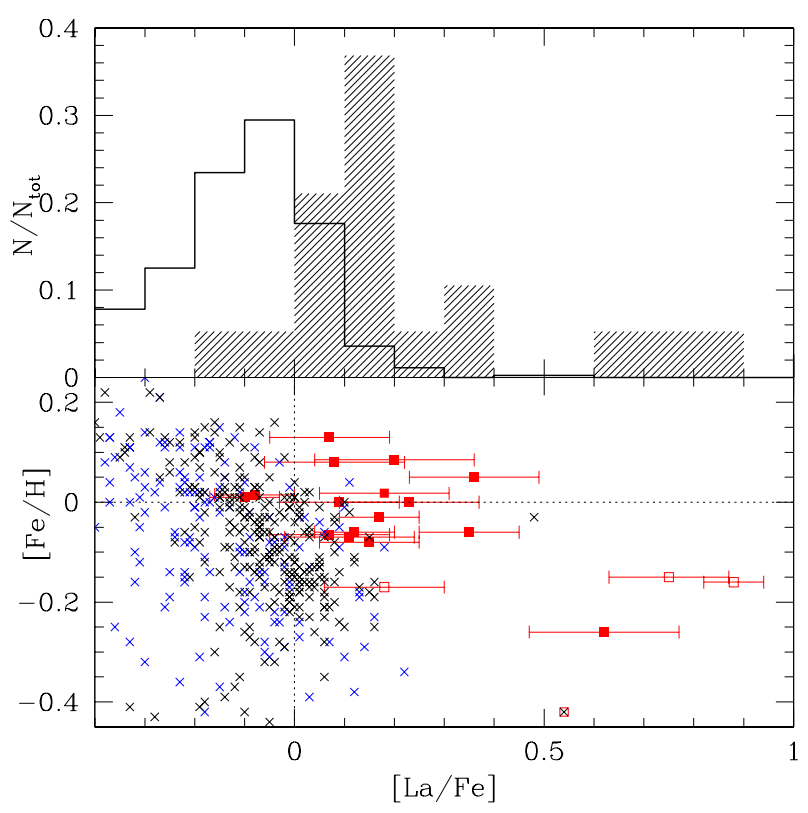

Fig. 13. Same as Fig. 12 for La.

may nevertheless be used to shed light on that question. The 12 giants from M07 subjected to an abundance study cover the range $-2.75 \leq \log Y \leq-2.05$ (Table 2). In that range, our statistical analysis of the reduced mass functions $Y$ predicts 21 systems with WD companions and 16 with main-sequence companions (see Fig. 2), or a percentage of $57 \%(=21 / 37)$ for the former ${ }^{3}$. Applied to the sample of 12 red giants whose $s$ process abundances were derived, this percentage would imply that $0.57 \times 12=6.8 \pm 1.4$ barium stars should have been found if all systems with WD companions were barium stars; the uncertainty on that value is estimated from the hypergeometric distribution, with $N=37, N_{1}=12$, and $p=X / N=21 / 37=0.57$. Only four were found, suggesting (despite the small-number statistics) that not all mass-transfer events from an AGB companion lead to the formation of a barium star.

Another signature of mass transfer is the blue straggler phenomenon. Geller \& Mathieu (2011), Gosnell et al. (2014), and Gosnell et al. (2015) have shown that (most of the) blue straggler stars in the cluster NGC 188 are binary systems, some with detected WD companions. The distribution of these binaries in the $(P-e)$ diagram is shown in Fig. 11, using the orbital elements from Gosnell et al. (2015). Surprisingly, considering the fact the blue stragglers are supposed to be post-mass-transfer objects, some of these objects are located outside the region defined by the post-mass-transfer barium stars. Unfortunately, a possible barium overabundance in the blue-straggler binaries of NGC 188 has not been tested, as was carried out by Milliman et al. (2015) for the blue stragglers in NGC 6819. In the latter cluster, five blue stragglers are found to be enriched in barium. Among these however, four show no sign of binarity and one is a SB2 system; neither situation is compatible with the mass-transfer scenario of matter rich in $s$-process elements from an AGB companion to produce such barium-rich stars. Overall, the relation between barium stars and blue stragglers has thus not yet been convincingly established.

3 This percentage is larger than the overall $22 \%$ fraction discussed earlier because of the pre-selected range in $\log Y$. 


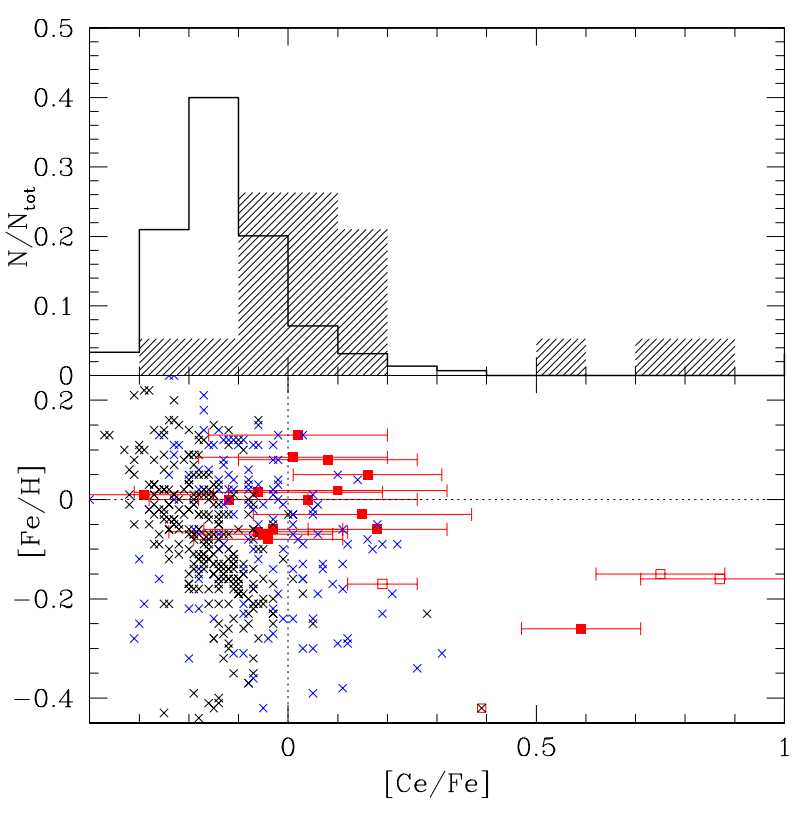

Fig. 14. Same as Fig. 12 for Ce.

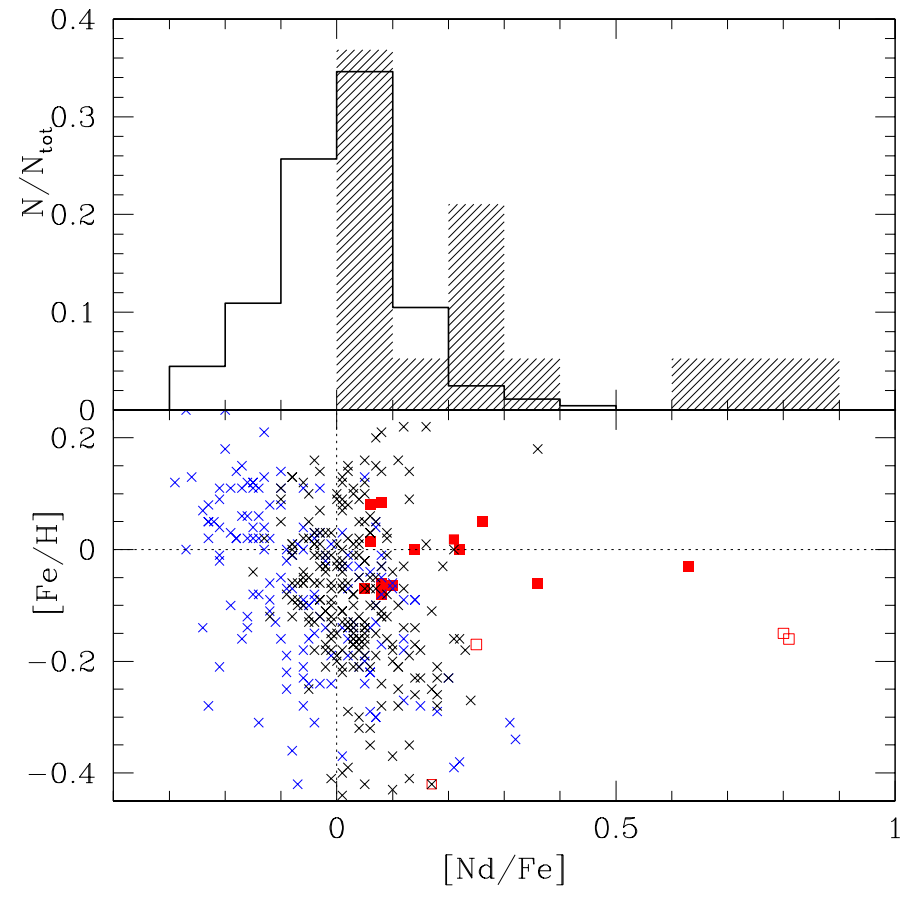

Fig. 15. Same as Fig. 12 for Nd. There is no error bar available on the $\mathrm{Nd}$ abundance, which is derived from a single line.

\section{Conclusions}

This paper presents a study of the mass-function distributions of red giants in open clusters and in barium systems. As far as the cluster sample is concerned, we conclude that $22 \%$ of the sample corresponds to post-mass-transfer systems. These systems must have WD companions. An abundance study of 12 cluster giant stars with a reduced mass function compatible with such a WD companion reveals that only 4 are indeed barium stars ( 3 are mild barium stars, and 1 is a strong barium star), whereas the statistics predicts 7 barium stars if all mass-transfer events ending up with a WD companion would also contaminate the accreting star with $s$-process elements and turn it into a barium star. We are thus led to conclude that this is not always the case. This lack of

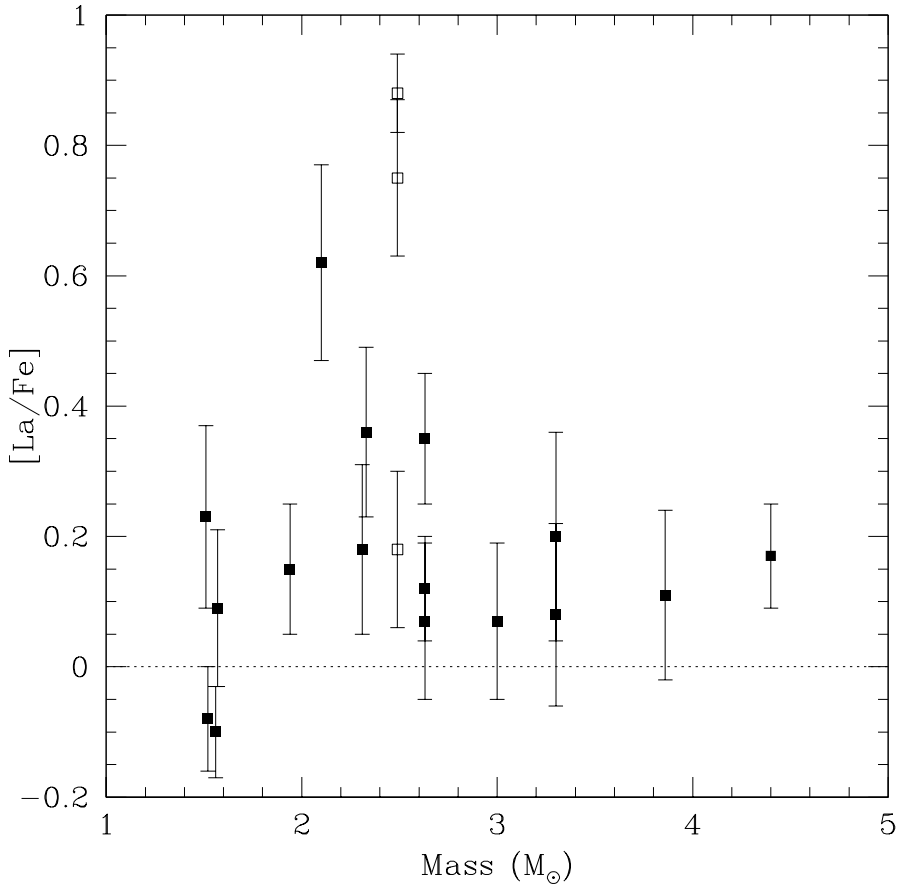

Fig. 16. Relation between $[\mathrm{La} / \mathrm{Fe}]$ and the mass of the giant, taken from Table A.4. Open symbols denote data taken from literature, from the reference listed in Table 2, and filled symbols denote abundances from the present analysis.

$s$-process contamination is probably related to the high metallicity of the considered clusters (most have solar, or even slightly super-solar metallicity), since all strong barium stars are found in the clusters with the lowest metallicities. This result confirms the larger efficiency of the $s$-process nucleosynthesis predicted at lower metallicities.

An important result of our analysis is the fact that some postmass-transfer systems from the M07 sample of giants in open clusters are located outside the locus of barium systems in the $(P-e)$ diagram. The same conclusion holds for the blue-straggler binaries in the cluster NGC 188. The origin of this difference in the dynamical outcome of mass transfer in barium stars, on one hand, and blue-straggler or open-cluster systems, on the other hand, is so far unknown.

Regarding barium stars, we confirm earlier results that their mass-function distribution is typical of WD companions, but in the absence of a reliable mass distribution for the barium giants, it is not possible to compare the WD mass distribution in barium systems with that of field WDs.

Acknowledgements. M.v.d.S. and S.v.E. are supported by a grant from the Fondation ULB. Based on observations obtained with the HERMES spectrograph, which is supported by the Research Foundation - Flanders (FWO), Belgium, the Research Council of KU Leuven, Belgium, the Fonds National de la Recherche Scientifique (F.R.S.-FNRS), Belgium, the Royal Observatory of Belgium, the Observatoire de Genève, Switzerland and the Thüringer Landessternwarte Tautenburg, Germany. We thank the anonymous referee for useful comments.

\section{References}

Alvarez, R., \& Plez, B. 1998, A\&A, 330, 1109

Baranne, A., Mayor, M., \& Poncet, J. L. 1979, Vistas Astron., 23, 279

Bessell, M. S., Castelli, F., \& Plez, B. 1998, A\&A, 333, 231

Bidelman, W. P., \& Keenan, P. C. 1951, ApJ, 114, 473

Boffin, H. M. J. 2010, A\&A, 524, A14 
Boffin, H. M. J. 2012, Orbital Couples: Pas de Deux in the Solar System and the Milky Way (Observatoire de Paris), 41

Boffin, H. M. J., \& Jorissen, A. 1988, A\&A, 205, 155

Boffin, H. M. J., Paulus, G., \& Cerf, N. 1992, in Binaries as Tracers of Star Formation, eds. A. Duquennoy, \& M. Mayor (Cambridge: Cambridge University Press), 26

Boffin, H. M. J., Cerf N., \& Paulus G. 1993, A\&A, 271, 125

Boffin, H. M. J., Hillen, M., Berger, J. P., et al. 2014, A\&A, 564, A1

Bonačić Marinović, A. A., Glebbeek, E., \& Pols, O. R. 2008, A\&A, 480, 797

Carquillat, J.-M., \& Prieur, J.-L. 2008, Astron. Nachr., 329, 44

Carquillat, J. M., Jorissen, A., Udry, S., \& Ginestet, N. 1998, A\&AS, 131, 49

Cerf, N., \& Boffin, H. M. J. 1994, Inverse Problems, 10, 533

Dermine, T., Izzard, R. G., Jorissen, A., \& Church, R. 2011, in Why Galaxie Care about AGB Stars II: Shining Examples and Common Inhabitants, eds. F. Kerschbaum, T. Lebzelter, \& R. F. Wing (San Francisco: ASP), ASP Conf Ser., 445,447

Duquennoy, A., \& Mayor, M. 1991, A\&A, 248, 485

Falcon, R. E., Winget, D. E., Montgomery, M. H., \& Williams, K. A. 2010, ApJ, 712,585

Frankowski, A., \& Jorissen, A. 2006, The Observatory, 126, 25

Geller, A. M., \& Mathieu, R. D. 2011, Nature, 478, 356

Goriely, S., \& Mowlavi, N. 2000, A\&A, 362, 599

Gorlova, N., Van Winckel, H., Vos, J., et al. 2013, EAS Pub. Ser., 64, 163

Gosnell, N. M., Mathieu, R. D., Geller, A. M., et al. 2014, ApJ, 783, L8

Gosnell, N. M., Mathieu, R. D., Geller, A. M., et al. 2015, ApJ, 814, 163

Griffin, R. F. 1984, The Observatory, 104, 224

Griffin, R. F. 1991, The Observatory, 111, 29

Griffin, R. F. 2006, The Observatory, 126, 1

Griffin, R. F. 2008, The Observatory, 128, 474

Griffin, R. F. 2009, The Observatory, 129, 6

Griffin, R., \& Griffin, R. 1980, MNRAS, 193, 957

Griffin, R. F., \& Keenan, P. C. 1992, The Observatory, 112, 168

Griffin, R. F., Jorissen, A., \& Mayor, M. 1996, The Observatory, 116, 298

Gustafsson, B., Edvardsson, B., Eriksson, K., et al. 2008, A\&A, 486, 951

Heiter, U., Lind, K., Asplund, M., et al. 2015, Phys. Scr., 90, 054010

Hurley, J. R., Pols, O. R., \& Tout, C. A. 2000, MNRAS, 315, 543

Izzard, R. G., Dermine, T., \& Church, R. P. 2010, A\&A, 523, A10

Jorissen, A. 2003, in Asymptotic giant branch stars, Astronomy and Astrophysic Library, eds. H. J. Habing, \& H. Olofsson (New York, Berlin: Springer), 461

Jorissen, A., \& Boffin, H. M. J. 1992, in Binaries as Tracers of Star Formation, eds. A. Duquennoy, \& M. Mayor (Cambridge: Cambridge University Press), 110

Jorissen, A., \& Mayor, M. 1988, A\&A, 198, 187

Jorissen, A., Hennen, O., Mayor, M., Bruch, A., \& Sterken, C. 1995, A\&A, 301, 707

Jorissen, A., Van Eck, S., Mayor, M., \& Udry, S. 1998, A\&A, 332, 877

Käppeler, F., Gallino, R., Bisterzo, S., \& Aoki, W. 2011, Rev. Mod. Phys., 83, 157

Katime Santrich, O. J., Pereira, C. B., \& de Castro, D. B. 2013, AJ, 146, 39

Kleinman, S. J., Kepler, S. O., Koester, D., et al. 2013, ApJS, 204, 5
Kupka, F., Piskunov, N., Ryabchikova, T. A., Stempels, H. C., \& Weiss, W. W. 1999, A\&AS, 138, 119

Kupka, F. G., Ryabchikova, T. A., Piskunov, N. E., Stempels, H. C., \& Weiss, W. W. 2000, Balt. Astron., 9, 590

Luck, R. E., \& Heiter, U. 2007, AJ, 133, 2464

Lucy, L. B. 1974, AJ, 79, 745

Mathieu, R. D. 1992, in Binaries as Tracers of Star Formation, eds. A Duquennoy, \& M. Mayor (Cambridge: Cambridge University Press), 155

McClure, R. D. 1983, ApJ, 268, 264

McClure, R. D., \& Woodsworth, A. W. 1990, ApJ, 352, 709

McClure, R. D., Fletcher, J. M., \& Nemec, J. M. 1980, ApJ, 238, L35

Mennessier, M. O., Luri, X., Figueras, F., et al. 1997, A\&A, 326, 722

Merle, T., Jorissen, A., Masseron, T., et al. 2014, A\&A, 567, A30

Merle, T., Jorissen, A., Van Eck, S., Masseron, T., \& Van Winckel, H. 2016, A\&A, 586, A151

Mermilliod, J.-C., \& Mayor, M. 2007, A\&A, 470, 919

Mermilliod, J.-C., Andersen, J., Latham, D. W., \& Mayor, M. 2007, A\&A, 473, 829

Milliman, K. E., Mathieu, R. D., \& Schuler, S. C. 2015, AJ, 150, 84

Mishenina, T. V., Gorbaneva, T. I., Bienaymé, O., et al. 2007, Astron. Rep., 51, 382

Morgan, W. W., Keenan, P. C., \& Kellman, E. 1943, An Atlas of Stellar Spectra with an Outline of Spectral Classification (Univerity of Chicago Press), 30

North, P. 2014, in Putting A Stars into Context: Evolution, Environment, and Related Stars, eds. G. Mathys, E. Griffin, O. Kochukhov, R. Monier, \& G. Wahlgren (Moscow: Publishing house Pero), 63

North, P., Jorissen, A., \& Mayor, M. 2000, The Carbon Star Phenomenon, 177, 269

Plez, B. 2012, Astrophysics Source Code Library, 5004

Pols, O. R., Karakas, A. I., Lattanzio, J. C., \& Tout, C. A. 2003, in Symbiotic Stars Probing Stellar Evolution, eds. R. L. M. Corradi, J. Mikolajewska, \& T. J. Mahoney (San Francisco: ASP Conf. Ser.), 303, 290

Pourbaix, D., Jancart, S., \& Boffin, H. M. J. 2004, RMxAC, 21, 265

Ramírez, I., \& Meléndez, J. 2005, ApJ, 626, 465

Raskin, G., van Winckel, H., Hensberge, H., et al. 2011, A\&A, 526, A69

Sales Silva, J. V., Peña Suárez, V. J., Katime Santrich, O. J., et al. 2014, AJ, 148, 83

Schindler, M., Linsky, J. L., Helfand, D. J., Stencel, R. E., \& Basri, G. S. 1982, ApJ, 263, 269

Stefanik, R. P., Torres, G., Latham, D. W., et al. 2011, AJ, 141, 144

Stetson, P. B., \& Pancino, E. 2008, PASP, 120, 1332

Udry, S., Jorissen, A., Mayor, M., \& Van Eck, S. 1998a, A\&AS, 131, 25

Udry, S., Mayor, M., Van Eck, S., et al. 1998b, A\&AS, 131, 43

Van der Swaelmen, M., Hill, V., Primas, F., \& Cole, A. A. 2013, A\&A, 560, A44

Van Eck, S., Jorissen, A., Udry, S., et al. 2000, A\&AS, 145, 51

Van Winckel, H., Jorissen, A., Gorlova, N., et al. 2010, It., 81, 1022

Warner, B. 1965, MNRAS, 129, 263

Webbink, R. F. 1988, in Critical Observations Versus Physical Models for Close Binary Systems, eds. K.-C. Leung, \& D. S. Zhai (New York: Gordon \& Breach), 403 
Appendix A: Mass functions, periods, and eccentricities for barium and $S$ stars

Since the review by Jorissen et al. (1998), several more orbits for barium and $\mathrm{S}$ stars have appeared in the literature. We therefore considered it useful to collect all orbits available in Tables A.1 (strong barium stars), A.2 (mild barium stars), and A.3 (S stars).

Table A.1. Mass functions for barium stars with strong chemical anomalies used in the current study, along with orbital periods and eccentricities.

\begin{tabular}{|c|c|c|c|c|}
\hline Name & Period (d) & $e$ & $f(M)\left(M_{\odot}\right)$ & Ref. \\
\hline 121447 & $185.7 \pm 0.1$ & $0.01 \pm 0.01$ & $0.025 \pm 0.001$ & 1 \\
\hline 120620 & $217.2 \pm 0.1$ & $0.01 \pm 0.01$ & $0.062 \pm 0.001$ & 4 \\
\hline$+38^{\circ} 118(1+2)$ & $299.4 \pm 0.2$ & $0.14 \pm 0.01$ & $0.014 \pm 0.0004$ & 4 \\
\hline 24035 & $377.8 \pm 0.3$ & $0.02 \pm 0.01$ & $0.047 \pm 0.003$ & 4 \\
\hline$-64^{\circ} 4333$ & $386.0 \pm 0.5$ & $0.03 \pm 0.01$ & $0.068 \pm 0.003$ & 4 \\
\hline 46407 & $457.4 \pm 0.1$ & $0.013 \pm 0.008$ & $0.035 \pm 0.001$ & 5 \\
\hline 100503 & $554.4 \pm 1.9$ & $0.06 \pm 0.05$ & $0.011 \pm 0.001$ & 4 \\
\hline 199939 & $584.9 \pm 0.7$ & $0.28 \pm 0.01$ & $0.025 \pm 0.001$ & 6 \\
\hline 44896 & $628.9 \pm 0.9$ & $0.02 \pm 0.01$ & $0.048 \pm 0.0015$ & 5 \\
\hline 92626 & $918.2 \pm 1.2$ & $0.00 \pm 0.01$ & $0.042 \pm 0.002$ & 5 \\
\hline Lu163 & $965 \pm 15$ & $0.03 \pm 0.07$ & $0.0029 \pm 0.0006$ & 4 \\
\hline 211594 & $1018.9 \pm 2.7$ & $0.06 \pm 0.01$ & $0.0140 \pm 0.0005$ & 5 \\
\hline 31487 & $1066.4 \pm 2.6$ & $0.05 \pm 0.01$ & $0.038 \pm 0.002$ & 6 \\
\hline NGC $2420-250(\mathrm{X})$ & $1403.6 \pm 3.5$ & $0.08 \pm 0.03$ & $0.047 \pm 0.005$ & 7 \\
\hline 88562 & $1445.0 \pm 8.5$ & $0.20 \pm 0.02$ & $0.048 \pm 0.003$ & 4 \\
\hline NGC 2420-173 & $1479 \pm 9$ & $0.43 \pm 0.05$ & $0.008 \pm 0.002$ & 7 \\
\hline 84678 & $1630 \pm 10$ & $0.06 \pm 0.02$ & $0.062 \pm 0.003$ & 4 \\
\hline 154430 & $1668 \pm 17$ & $0.11 \pm 0.03$ & $0.034 \pm 0.003$ & 4 \\
\hline 43389 & $1689 \pm 9$ & $0.08 \pm 0.02$ & $0.043 \pm 0.002$ & 5 \\
\hline 101013 & $1711 \pm 4$ & $0.20 \pm 0.01$ & $0.037 \pm 0.001$ & 6,8 \\
\hline 201657 & $1710 \pm 15$ & $0.17 \pm 0.07$ & $0.004 \pm 0.001$ & 5 \\
\hline 49641 & $1768 \pm 23$ & $0.07 \pm 0.11$ & $0.0031 \pm 0.0004$ & 6,21 \\
\hline 5424 & $1882 \pm 19$ & $0.23 \pm 0.04$ & $0.005 \pm 0.0004$ & 4 \\
\hline 16458 & $2018 \pm 12$ & $0.10 \pm 0.02$ & $0.041 \pm 0.003$ & 1 \\
\hline 20394 & $2226 \pm 22$ & $0.20 \pm 0.03$ & $0.0020 \pm 0.0005$ & 9 \\
\hline 36598 & $2653 \pm 23$ & $0.08 \pm 0.02$ & $0.037 \pm 0.002$ & 4 \\
\hline 178717 & $2866 \pm 21$ & $0.43 \pm 0.03$ & $0.006 \pm 0.001$ & 6 \\
\hline 201824 & $2837 \pm 13$ & $0.34 \pm 0.02$ & $0.040 \pm 0.003$ & 9 \\
\hline 50082 & $2896 \pm 21$ & $0.19 \pm 0.02$ & $0.027 \pm 0.002$ & 5 \\
\hline 42537 & $3216 \pm 55$ & $0.16 \pm 0.05$ & $0.027 \pm 0.005$ & 4 \\
\hline$-42^{\circ} 2048$ & $3260 \pm 28$ & $0.08 \pm 0.02$ & $0.065 \pm 0.004$ & 4 \\
\hline 107541 & $3570 \pm 46$ & $0.10 \pm 0.03$ & $0.029 \pm 0.002$ & 5 \\
\hline$+38^{\circ} 118(12+3)$ & $3877 \pm 112$ & $0.21 \pm 0.06$ & $0.0017 \pm 0.0004$ & 4 \\
\hline 196445 & $3221 \pm 43$ & $0.24 \pm 0.02$ & $0.031 \pm 0.002$ & 4 \\
\hline 60197 & $3244 \pm 66$ & $0.34 \pm 0.05$ & $0.0028 \pm 0.0006$ & 4 \\
\hline 123949 & $8539 \pm 25$ & $0.88 \pm 0.01$ & $0.048 \pm 0.007$ & 0 \\
\hline 211954 & $10908 \pm 164$ & $0.24 \pm 0.06$ & $0.076 \pm 0.071$ & 0 \\
\hline 65854 & $\mathrm{SB}$ & & & 0 \\
\hline 19014 & cst? & & & 10 \\
\hline
\end{tabular}

Notes. The systems are ordered by increasing orbital period. References are provided at the end of Table A.3. If not specified otherwise, the column "Name" lists the HD number. 
M. Van der Swaelmen et al.: Orbital properties of barium and S stars, and red giants in open clusters

Table A.2. Same as Table A.1 for Ba stars with mild chemical anomalies.

\begin{tabular}{|c|c|c|c|c|}
\hline Name & Period (d) & $e$ & $f(M)\left(M_{\odot}\right)$ & Ref. \\
\hline 77247 & $80.53 \pm 0.01$ & $0.09 \pm 0.01$ & $0.0050 \pm 0.0001$ & 6 \\
\hline 218356 & $111.16 \pm 0.02$ & 0 & $0.000037 \pm 0.000003$ & 11 \\
\hline 136138 & $506.4 \pm 0.2$ & $0.333 \pm 0.006$ & $0.0113 \pm 0.0003$ & 3, 14 (HR 5692) \\
\hline 58368 & $672.7 \pm 1.3$ & $0.22 \pm 0.02$ & $0.021 \pm 0.001$ & 6 \\
\hline 49841 & $896 \pm 2$ & $0.15 \pm 0.02$ & $0.032 \pm 0.002$ & 5 \\
\hline 58121 & $1214.3 \pm 5.7$ & $0.14 \pm 0.02$ & $0.015 \pm 0.001$ & 5 \\
\hline 26886 & $1263.2 \pm 3.7$ & $0.39 \pm 0.02$ & $0.025 \pm 0.002$ & 5 \\
\hline 223617 & $1293.7 \pm 3.9$ & $0.06 \pm 0.02$ & $0.0064 \pm 0.0004$ & 5,6 \\
\hline 143899 & $1461.6 \pm 6.9$ & $0.19 \pm 0.02$ & $0.017 \pm 0.001$ & 4 \\
\hline 210946 & $1529.5 \pm 4.1$ & $0.13 \pm 0.01$ & $0.041 \pm 0.001$ & 5 \\
\hline 101079 & $1563 \pm 2$ & $0.171 \pm 0.005$ & $0.00232 \pm 0.00005$ & 0 \\
\hline 95193 & $1653.7 \pm 9.0$ & $0.13 \pm 0.02$ & $0.026 \pm 0.001$ & 4 \\
\hline 27271 & $1693.8 \pm 9.1$ & $0.22 \pm 0.02$ & $0.024 \pm 0.001$ & 5 \\
\hline 200063 & $1735.4 \pm 8.1$ & $0.07 \pm 0.04$ & $0.058 \pm 0.004$ & 5 \\
\hline 91208 & $1754 \pm 13$ & $0.17 \pm 0.02$ & $0.022 \pm 0.002$ & 4 \\
\hline 288174 & $1817.5 \pm 6.7$ & $0.20 \pm 0.01$ & $0.017 \pm 0.001$ & 4 \\
\hline 204075 & $2378 \pm 55$ & $0.28 \pm 0.07$ & $0.004 \pm 0.001$ & 6,10 \\
\hline 205011 & $2837 \pm 10$ & $0.24 \pm 0.02$ & $0.034 \pm 0.003$ & 6,10 \\
\hline 131670 & $2930 \pm 12$ & $0.16 \pm 0.01$ & $0.040 \pm 0.002$ & 4,6 \\
\hline$-01^{\circ} 3022$ & $3253 \pm 31$ & $0.28 \pm 0.02$ & $0.016 \pm 0.001$ & 4 \\
\hline$-14^{\circ} 2678$ & $3470 \pm 107$ & $0.22 \pm 0.04$ & $0.023 \pm 0.003$ & 4 \\
\hline 59852 & $3464 \pm 54$ & $0.15 \pm 0.06$ & $0.0022 \pm 0.0004$ & 4 \\
\hline 180622 & $4049 \pm 38$ & $0.06 \pm 0.10$ & $0.07 \pm 0.02$ & 0 \\
\hline 216219 & $4098 \pm 111$ & $0.10 \pm 0.04$ & $0.013 \pm 0.001$ & 5 \\
\hline 183915 & $4341 \pm 25$ & $0.47 \pm 0.06$ & $0.00009 \pm 0.00004$ & 0 \\
\hline 165141 & $4760 \pm 120$ & $0.05 \pm 0.03$ & $0.015 \pm 0.001$ & 20 \\
\hline$-10^{\circ} 4311$ & 4888. \pm 14 & $0.078 \pm 0.007$ & $0.062 \pm 0.004$ & 0 \\
\hline 199394 & $5205 \pm 7$ & $0.14 \pm 0.01$ & $0.039 \pm 0.001$ & 0,22 \\
\hline 139195 & 5324. \pm 19 & $0.35 \pm 0.02$ & $0.026 \pm 0.002$ & 12 \\
\hline 40430 & $5570:$ & 0.25 & - & 0 \\
\hline 22589 & $5761 \pm 88$ & $0.28 \pm 0.03$ & $0.0030 \pm 0.0004$ & 0 \\
\hline 202109 & $6489 \pm 31$ & $0.22 \pm 0.03$ & $0.023 \pm 0.003$ & 13 \\
\hline 196673 & $7636 \pm 153$ & $0.66 \pm 0.03$ & $0.019 \pm 0.006$ & 0 \\
\hline 18182 & 8056: & $0.3:$ & - & 0 \\
\hline 53199 & $8300 . \pm 99$ & $0.24 \pm 0.01$ & $0.029 \pm 0.003$ & 0 \\
\hline 134698 & 9386: & $0.91:$ & - & 0 \\
\hline 51959 & 9488: & $0.58:$ & - & 0 \\
\hline 104979 & $13940:$ & 0.2 & - & 0 \\
\hline 98839 & $16419 \pm 116$ & $0.556 \pm 0.006$ & $0.058 \pm 0.004$ & 0 \\
\hline 119185 & 19467: & $0.4:$ & - & 0 \\
\hline 50843 & SB & & & 0 \\
\hline 95345 & cst? & & & 0 \\
\hline
\end{tabular}


Table A.3. Same as Table A.1 for S (and C) stars.

\begin{tabular}{|c|c|c|c|c|}
\hline Name (HD/DM) & Period (d) & $e$ & $f(M)\left(M_{\odot}\right)$ & Ref. \\
\hline & & & \multicolumn{2}{|l|}{ Extrinsic S stars } \\
\hline 121447 & $185.7 \pm 0.1$ & $0.01 \pm 0.01$ & $0.025 \pm 0.001$ & 1 \\
\hline 95875 & $197.2 \pm 0.3$ & 0.0 & $0.061 \pm 0.007$ & 19 (Hen4-108) \\
\hline$-25^{\circ} 10393$ & $346.6 \pm 0.03$ & $0.104 \pm 0.003$ & $0.066 \pm 0.001$ & 0, 19 (Hen 4-147) \\
\hline 191589 & $377.3 \pm 0.1$ & $0.250 \pm 0.003$ & $0.394 \pm 0.005$ & 4 \\
\hline 189581 & $614 \pm 1$ & $0.39 \pm 0.02$ & $(1.3 \pm 0.1) \times 10^{-5}$ & 0 \\
\hline 22649 & $596.2 \pm 0.2$ & $0.09 \pm 0.02$ & $0.037 \pm 0.003$ & 15 \\
\hline 35155 & $638.2 \pm 0.3$ & $0.07 \pm 0.03$ & $0.032 \pm 0.003$ & 16 (V1261 Ori) \\
\hline 332077 & $669.1 \pm 1.0$ & $0.077 \pm 0.007$ & $1.25 \pm 0.02$ & 10 \\
\hline$+24^{\circ} 620$ & $773.4 \pm 5.5$ & $0.06 \pm 0.03$ & $0.042 \pm 0.005$ & 10 \\
\hline$+22^{\circ} 700$ & $849.5 \pm 8.8$ & $0.08 \pm 0.06$ & $0.043 \pm 0.008$ & 10 \\
\hline$+23^{\circ} 3093$ & $1008.1 \pm 4.8$ & $0.39 \pm 0.03$ & $0.045 \pm 0.005$ & 10 \\
\hline 215336 & $1143.3 \pm 0.6$ & $0.021 \pm 0.003$ & $0.039 \pm 0.003$ & 0 \\
\hline 9810 & $1147 \pm 2$ & $0.21 \pm 0.01$ & $0.019 \pm 0.001$ & 19 (Hen 4-2) \\
\hline 63733 & $1160.7 \pm 8.9$ & $0.23 \pm 0.03$ & $0.025 \pm 0.003$ & 4 \\
\hline 191226 & $1210.4 \pm 4.3$ & $0.19 \pm 0.02$ & $0.013 \pm 0.001$ & 17 \\
\hline$+28^{\circ} 4592$ & $1252.9 \pm 3.5$ & $0.09 \pm 0.02$ & $0.016 \pm 0.001$ & 4 \\
\hline 246818 & $2548.5 \pm 73.2$ & $0.18 \pm 0.11$ & $0.004 \pm 0.002$ & 4 \\
\hline 49368 & $2996 \pm 67$ & $0.36 \pm 0.05$ & $0.022 \pm 0.003$ & 4 \\
\hline$+23^{\circ} 3992$ & $3096 \pm 42$ & $0.10 \pm 0.03$ & $0.034 \pm 0.004$ & 4 \\
\hline 343486 & $3166 \pm 38$ & $0.24 \pm 0.03$ & $0.039 \pm 0.005$ & 4 \\
\hline$+21^{\circ} 255(\mathrm{~S})$ & $4137 \pm 317$ & $0.21 \pm 0.04$ & $0.032 \pm 0.004$ & 4 \\
\hline 170970 & $4651 \pm 10$ & $0.19 \pm 0.01$ & $0.0213 \pm 0.0007$ & 0 \\
\hline$+31^{\circ} 4391$ & $6757 \pm 37$ & $0.14 \pm 0.03$ & $0.03 \pm 0.01$ & 0 \\
\hline$+79^{\circ} 156$ & $11119 \pm 69$ & $0.44 \pm 0.01$ & $0.022 \pm 0.002$ & 0 \\
\hline 184185 & 17490: & $0.37:$ & - & 0 \\
\hline 288833 & 17565: & $0.42:$ & - & 0 \\
\hline \multirow[t]{2}{*}{218634} & 90359: & 0.55 & - & 0 \\
\hline & & & \multicolumn{2}{|c|}{ Symbiotic S and C stars } \\
\hline$-28^{\circ} 3719$ & $399.1 \pm 0.1$ & $<0.002$ & $0.020 \pm 0.001$ & 0 (Hen 4-18) \\
\hline V420 Нya & $750.0 \pm 0.2$ & $0.092 \pm 0.002$ & $0.0928 \pm 0.0004$ & 0, 19 (Hen 4-121) \\
\hline 59643 & $1305 \pm 4$ & $0.18 \pm 0.03$ & $0.027 \pm 0.003$ & $18(\mathrm{C})$ \\
\hline ER Del & $2056 \pm 2$ & $0.233 \pm 0.004$ & $0.069 \pm 0.001$ & 16 \\
\hline 7351 & $4559 \pm 9$ & $0.11 \pm 0.01$ & $0.062 \pm 0.001$ & 0 \\
\hline
\end{tabular}

References. (To Tables A.1-A.3:) (0) Jorissen et al. (in prep.); (1) Jorissen et al. (1995); (2) Griffin (2008); (3) Stefanik et al. (2011); (4) Udry et al. (1998a); (5) Udry et al. (1998b); (6) McClure \& Woodsworth (1990); (7) Mermilliod et al. (2007); (8) Griffin \& Griffin (1980); (9) Griffin et al. (1996); (10) Jorissen et al. (1998); (11) Griffin (2006); (12) Griffin (1991); (13) Griffin \& Keenan (1992); (14) Griffin (2009); (15) Griffin (1984); (16) Boffin et al. (2014); (17) Carquillat et al. (1998); (18) Carquillat \& Prieur (2008); (19) Van Eck et al. (2000); (20) Fekel (priv. comm.); (21) a new orbital solution has been computed from McClure \& Woodsworth (1990) data, and the non-zero eccentricity has been adopted from that new computation (despite being compatible with a circular orbit); (22) a solution with $P=10480 \mathrm{~d}, e=0.36$, and $f(M)=0.11 \pm 0.01 M_{\odot}$ is also possible, although less probable given the large value of the mass function. 
M. Van der Swaelmen et al.: Orbital properties of barium and S stars, and red giants in open clusters

Table A.4. Open cluster stars and derived masses.

\begin{tabular}{|c|c|c|c|c|}
\hline Open cluster & Star & $\begin{array}{l}f(m) \\
\left(M_{\odot}\right) \\
\end{array}$ & $\begin{array}{l}\text { Mass } \\
\left(M_{\odot}\right)\end{array}$ & $\begin{array}{l}\text { Sigma } \\
\left(M_{\odot}\right)\end{array}$ \\
\hline IC 4651 & 6686 & 0.060700 & 2.050 & 0.05 \\
\hline IC 4651 & 8665 & 0.290000 & 2.050 & 0.05 \\
\hline IC 4651 & 10195 & 0.010380 & 2.050 & 0.05 \\
\hline IC 4651 & 14290 & 0.142000 & 2.050 & 0.05 \\
\hline IC 4651 & 14641 & 0.004820 & 2.050 & 0.05 \\
\hline IC 4725 & 150 & 0.349000 & 5.47 & 0.05 \\
\hline IC 4756 & 69 & 0.023700 & 2.630 & 0.05 \\
\hline IC 4756 & 80 & 0.203000 & 2.630 & 0.05 \\
\hline IC 4756 & 139 & 0.006450 & 2.630 & 0.05 \\
\hline Me 125 & 41 & 0.001010 & 2.480 & 0.05 \\
\hline Me 125 & 71 & 0.170000 & 2.480 & 0.05 \\
\hline Me 171 & 107 & 0.399000 & 3.467 & 0.05 \\
\hline Me 171 & 110 & 0.255400 & 3.467 & 0.05 \\
\hline Me 171 & 118 & 0.231700 & 3.467 & 0.05 \\
\hline Me 171 & 151 & 0.167300 & 3.467 & 0.05 \\
\hline Me 1105 & 17 & 0.040000 & 3.800 & 0.05 \\
\hline Me 1111 & 91 & 0.260000 & 2.760 & 0.05 \\
\hline NGC 0129 & 170 & 0.914000 & 5.90 & 0.05 \\
\hline NGC 0129 & 200 & 0.267500 & 5.90 & 0.05 \\
\hline NGC 0752 & 75 & 0.090300 & 1.940 & 0.05 \\
\hline NGC 0752 & 110 & 0.218600 & 1.940 & 0.05 \\
\hline NGC 0752 & 208 & 0.080400 & 1.940 & 0.05 \\
\hline NGC 1027 & 27 & 0.079600 & 4.330 & 0.11 \\
\hline NGC 1528 & 4 & 0.907000 & 2.978 & 0.05 \\
\hline NGC 1778 & 2 & 0.001210 & 3.000 & 0.05 \\
\hline NGC 1817 & 44 & 0.065400 & 2.770 & 0.05 \\
\hline NGC 1817 & 56 & 0.011700 & 2.770 & 0.05 \\
\hline NGC 1817 & 164 & 0.305400 & 2.764 & 0.05 \\
\hline NGC 1817 & 244 & 0.000440 & 2.764 & 0.05 \\
\hline NGC 2099 & 49 & 0.040100 & 3.300 & 0.05 \\
\hline NGC 2099 & 149 & 0.011040 & 3.300 & 0.05 \\
\hline NGC 2099 & 485 & 0.188400 & 3.350 & 0.05 \\
\hline NGC 2099 & 748 & 0.560000 & 3.084 & 0.05 \\
\hline NGC 2099 & 782 & 0.011200 & 3.120 & 0.05 \\
\hline NGC 2099 & 966 & 0.011 & 3.05 & 0.05 \\
\hline NGC 2215 & 26 & 0.023000 & 3.585 & 0.05 \\
\hline NGC 2287 & 21 & 0.016400 & 3.803 & 0.05 \\
\hline NGC 2287 & 97 & 0.000027 & 3.795 & 0.05 \\
\hline NGC 2287 & 102 & 0.670000 & 3.541 & 0.05 \\
\hline NGC 2287 & 107 & 0.037800 & 3.795 & 0.05 \\
\hline NGC 2324 & 1006 & 0.192000 & 2.700 & 0.1 \\
\hline NGC 2335 & 4 & 0.015250 & 4.400 & 0.05 \\
\hline NGC 2360 & 44 & 0.185000 & 2.600 & 0.13 \\
\hline NGC 2360 & 51 & 0.072400 & 2.730 & 0.05 \\
\hline NGC 2360 & 52 & 0.039800 & 2.480 & 0.05 \\
\hline NGC 2360 & 62 & 0.011900 & 2.480 & 0.05 \\
\hline NGC 2360 & 181 & 0.474000 & 2.600 & 0.13 \\
\hline NGC 2420 & 173 & 0.007900 & 2.096 & 0.05 \\
\hline NGC 2420 & 250 & 0.047300 & 2.096 & 0.05 \\
\hline NGC 2423 & 43 & 0.000850 & 2.320 & 0.05 \\
\hline NGC 2437 & 29 & 0.018900 & 3.535 & 0.05 \\
\hline NGC 2437 & 242 & 0.322300 & 3.588 & 0.05 \\
\hline NGC 2447 & 25 & 0.208900 & 2.926 & 0.05 \\
\hline NGC 2447 & 42 & 0.041000 & 2.926 & 0.05 \\
\hline
\end{tabular}


Table A.4. continued.

\begin{tabular}{|c|c|c|c|c|}
\hline Open cluster & Star & $\begin{array}{l}f(m) \\
\left(M_{\odot}\right)\end{array}$ & $\begin{array}{l}\text { Mass } \\
\left(M_{\odot}\right)\end{array}$ & $\begin{array}{l}\text { Sigma } \\
\left(M_{\odot}\right)\end{array}$ \\
\hline NGC 2477 & 1025 & 0.006980 & 2.330 & 0.05 \\
\hline NGC 2477 & 1044 & 0.010700 & 2.330 & 0.05 \\
\hline NGC 2477 & 1272 & 0.000048 & 2.330 & 0.05 \\
\hline NGC 2477 & 2064 & 0.081900 & 2.330 & 0.05 \\
\hline NGC 2477 & 2204 & 0.545000 & 2.330 & 0.05 \\
\hline NGC 2477 & 3003 & 0.209000 & 2.330 & 0.05 \\
\hline NGC 2477 & 3170 & 0.013500 & 2.330 & 0.05 \\
\hline NGC 2477 & 3176 & 0.012860 & 2.330 & 0.05 \\
\hline NGC 2477 & 4067 & 0.000220 & 2.330 & 0.05 \\
\hline NGC 2477 & 4137 & 0.095200 & 2.330 & 0.05 \\
\hline NGC 2477 & 5073 & 0.057900 & 2.330 & 0.05 \\
\hline NGC 2477 & 6020 & 0.015400 & 2.330 & 0.05 \\
\hline NGC 2477 & 6062 & 0.011800 & 2.330 & 0.05 \\
\hline NGC 2477 & 6251 & 0.000300 & 2.330 & 0.05 \\
\hline NGC 2477 & 8017 & 0.064300 & 2.330 & 0.05 \\
\hline NGC 2477 & 8018 & 0.004010 & 2.330 & 0.05 \\
\hline NGC 2482 & 23 & 0.071700 & 3.000 & 0.10 \\
\hline NGC 2533 & 17 & 0.001940 & 2.400 & 0.125 \\
\hline NGC 2539 & 114 & 0.012400 & 3.000 & 0.05 \\
\hline NGC 2539 & 209 & 0.017200 & 3.000 & 0.05 \\
\hline NGC 2539 & 209 & 0.015400 & 3.000 & 0.05 \\
\hline NGC 2539 & 223 & 0.351000 & 3.000 & 0.05 \\
\hline NGC 2539 & 233 & 0.000013 & 3.000 & 0.05 \\
\hline NGC 2539 & 663 & 0.001170 & 3.000 & 0.05 \\
\hline NGC 2548 & 1296 & 0.007900 & 3.035 & 0.05 \\
\hline NGC 2548 & 1560 & 0.547000 & 3.150 & 0.15 \\
\hline NGC 2567 & 104 & 0.280300 & 3.330 & 0.08 \\
\hline NGC 2632 & 428 & 0.018980 & 2.410 & 0.08 \\
\hline NGC 2682 & 136 & 0.002300 & 1.502 & 0.05 \\
\hline NGC 2682 & 143 & 0.002680 & 1.511 & 0.05 \\
\hline NGC 2682 & 170 & 0.005900 & 1.555 & 0.05 \\
\hline NGC 2682 & 224 & 0.142000 & 1.567 & 0.05 \\
\hline NGC 2682 & 244 & 0.009100 & 1.522 & 0.05 \\
\hline NGC 2972 & 14 & 0.008120 & 5.450 & 0.11 \\
\hline NGC 3532 & 152 & 0.172800 & 3.187 & 0.05 \\
\hline NGC 3532 & 160 & 0.010030 & 3.330 & 0.05 \\
\hline NGC 3680 & 27 & 0.006100 & 2.000 & 0.10 \\
\hline NGC 3960 & 50 & 0.070800 & 2.370 & 0.05 \\
\hline NGC 3960 & 91 & 0.030100 & 2.370 & 0.05 \\
\hline NGC 3960 & 275 & 0.067600 & 2.45 & 0.13 \\
\hline NGC 4349 & 79 & 0.085200 & 3.860 & 0.08 \\
\hline NGC 4349 & 203 & 0.016510 & 3.860 & 0.08 \\
\hline NGC 5822 & 2 & 0.009160 & 2.490 & 0.11 \\
\hline NGC 5822 & 3 & 0.023000 & 2.490 & 0.11 \\
\hline NGC 5822 & 4 & 0.024200 & 2.490 & 0.11 \\
\hline NGC 5822 & 11 & 0.039400 & 2.490 & 0.11 \\
\hline NGC 5822 & 80 & 0.087430 & 2.490 & 0.11 \\
\hline NGC 5822 & 151 & 0.011700 & 2.490 & 0.11 \\
\hline NGC 5822 & 276 & 0.004600 & 2.490 & 0.11 \\
\hline NGC 5822 & 312 & 0.196000 & 2.490 & 0.11 \\
\hline NGC 5823 & 1034 & 0.288000 & 2.200 & 0.20 \\
\hline NGC 6124 & 29 & 0.006260 & 4.570 & 0.11 \\
\hline NGC 6124 & 33 & 0.358000 & 4.570 & 0.11 \\
\hline
\end{tabular}


Table A.4. continued.

\begin{tabular}{lrccl}
\hline \hline Open cluster & Star & $\begin{array}{c}f(m) \\
\left(M_{\odot}\right)\end{array}$ & $\begin{array}{c}\text { Mass } \\
\left(M_{\odot}\right)\end{array}$ & $\begin{array}{l}\text { Sigma } \\
\left(M_{\odot}\right)\end{array}$ \\
\hline NGC 6134 & 8 & 0.034100 & 2.250 & 0.11 \\
NGC 6134 & 34 & 0.014200 & 2.250 & 0.11 \\
NGC 6134 & 204 & 0.005310 & 2.250 & 0.11 \\
NGC 6192 & 96 & 0.000550 & 4.53 & 0.05 \\
NGC 6475 & 58 & 0.025600 & 3.500 & 0.05 \\
NGC 6475 & 134 & 0.017990 & 3.500 & 0.05 \\
NGC 6633 & 70 & 0.807000 & 2.820 & 0.05 \\
NGC 6694 & 14 & 0.001080 & 5.560 & 0.05 \\
NGC 6705 & 926 & 0.750000 & 3.840 & 0.05 \\
NGC 6705 & 1223 & 0.430000 & 3.840 & 0.05 \\
NGC 6709 & 303 & 0.150800 & 4.430 & 0.11 \\
NGC 6940 & 84 & 0.031100 & 2.308 & 0.05 \\
NGC 6940 & 92 & 0.005300 & 2.312 & 0.05 \\
NGC 6940 & 100 & 0.181200 & 2.306 & 0.05 \\
NGC 6940 & 111 & 0.020500 & 2.312 & 0.05 \\
NGC 6940 & 130 & 0.027600 & 2.312 & 0.05 \\
NGC 6940 & 189 & 0.280000 & 2.308 & 0.05 \\
NGC 7209 & 95 & 0.018130 & 2.980 & 0.13 \\
\hline
\end{tabular}

\section{Appendix B: Chemical analysis}

\section{B.1. Stellar parameters}

The effective temperature $T_{\text {eff }}$ was derived from the de-reddened $(V-K)_{0}$ index, using the photometric data listed in the WEBDA database, and the calibration of Bessell et al. (1998), which also provided the bolometric correction. The surface gravity $\log g$ was derived from its definition $g=G M / R^{2}$, with $G$ the gravitational constant, $M$ the stellar mass (taken from Table A.4), and $R$ the stellar radius, derived from the Stefan-Boltzmann relation, using $T_{\text {eff }}$ as above, and $L$ from the cluster distance modulus and bolometric magnitude. The cluster metallicity, as listed in Table 2, is adopted from the WEBDA database. The microturbulence velocity $v_{\text {turb }}$ was set at $1.5 \mathrm{~km} \mathrm{~s}^{-1}$ for all stars.

\section{B.2. Line list}

We compiled the atomic line list from the line database VALD 4 (Kupka et al. 1999, 2000). Molecular transitions from ${ }^{12} \mathrm{C}^{14} \mathrm{~N}$ and ${ }^{13} \mathrm{C}^{14} \mathrm{~N}$ (Plez, priv. comm.) were also included in the computation of synthetic spectra. We chose the lines to be measured following the prescription by Van der Swaelmen et al. (2013), Merle et al. (2014), and Merle et al. (2016). We took into account the hyperfine structure for the following lines: Ba II at $585.3668 \mathrm{~nm}, 614.1711 \mathrm{~nm}$, and $649.6900 \mathrm{~nm}$, and La II at

\footnotetext{
4 http://vald.astro.univie.ac.at/ vald/php/vald.php
}

$626.2422 \mathrm{~nm}$, and $639.0477 \mathrm{~nm}$. Table B.1 provides the final list of lines used in this paper.

\section{B.3. Abundance determination}

We use spectral synthesis, as described in Van der Swaelmen et al. (2013), to derive the chemical abundances of the $s$-elements $\mathrm{Y}, \mathrm{Zr}, \mathrm{La}, \mathrm{Ce}$, and $\mathrm{Nd}$. To this end, a grid of theoretical spectra is computed with turbospectrum (Alvarez \& Plez 1998; Plez 2012), while stellar-atmosphere models are interpolated from the grid of MARCS (spherical) model atmospheres ${ }^{5}$ (Gustafsson et al. 2008).

The radiative transfer is computed at local thermodynamical equilibrium (LTE) in spherical geometry. As described in Van der Swaelmen et al. (2013), the $\chi^{2}$ algorithm selecting the best-fit model weights each wavelength by the total flux of the contaminating species, such that the more contaminated a pixel is, the less it counts in the $\chi^{2}$. The contaminating flux is obtained from a synthetic spectrum where the line under consideration has been suppressed, thus revealing the contribution from all other species. Figure B.1 shows the fits to the observed spectrum of the star IC 4756.69. Tables 3 and B.2 give averaged and line-byline abundances for the target stars.

\section{B.4. Error budget}

Table 3 gives the standard deviation around the mean abundance ratios, for each ion with at least three independently measured lines. Since we are dealing with small samples, an estimate of the

\footnotetext{
5 models available at http : //marcs.astro.uu.se/
} 
Table B.1. List of measured lines.

\begin{tabular}{|c|c|c|c|c|}
\hline Species & $\begin{array}{c}\lambda \\
(\AA)\end{array}$ & $\begin{array}{l}\chi \text { exc } \\
(\mathrm{eV})\end{array}$ & $\log g f_{\text {adopted }}$ & \\
\hline Y II & 4883.682 & 1.084 & 0.000 & $\mathrm{c}$ \\
\hline Y II & 5087.416 & 1.084 & -0.330 & $\mathrm{c}$ \\
\hline Y II & 5200.406 & 0.992 & -0.730 & c \\
\hline Y II & 5289.815 & 1.033 & -1.900 & $\mathrm{c}$ \\
\hline Y II & 5320.782 & 1.084 & -1.950 & d \\
\hline Y II & 5402.774 & 1.839 & -0.640 & $\mathrm{c}$ \\
\hline Y II & 5544.611 & 1.738 & -0.980 & c \\
\hline Y II & 5546.009 & 1.748 & -1.120 & $\mathrm{c}$ \\
\hline Y II & 5728.887 & 1.839 & -1.300 & $\mathrm{c}$ \\
\hline Y II & 6795.414 & 1.738 & -1.620 & c \\
\hline $\mathrm{ZrI}$ & 4772.310 & 0.623 & 0.040 & d \\
\hline $\mathrm{ZrI}$ & 6127.440 & 0.154 & -1.060 & d \\
\hline $\mathrm{ZrI}$ & 6134.550 & 0.000 & -1.280 & d \\
\hline $\mathrm{ZrI}$ & 6143.200 & 0.071 & -1.100 & d \\
\hline Zr II & 5112.270 & 1.665 & -0.850 & d \\
\hline La II & 4558.460 & 0.321 & -0.970 & d \\
\hline La II & 4574.860 & 0.173 & -1.080 & d \\
\hline La II & 4662.500 & 0.000 & -1.240 & $\mathrm{~d}$ \\
\hline La II & 4748.730 & 0.927 & -0.540 & d \\
\hline La II & $4804.039 *$ & 0.235 & -1.490 & $\mathrm{~b}$ \\
\hline La II & 4920.980 & 0.126 & -0.580 & d \\
\hline La II & 5114.560 & 0.235 & -1.030 & d \\
\hline La II & 5290.820 & 0.000 & -1.650 & d \\
\hline La II & $5303.528 *$ & 0.321 & -1.350 & b \\
\hline La II & 5797.570 & 0.244 & -1.360 & d \\
\hline La II & 5880.630 & 0.235 & -1.830 & d \\
\hline La II & $6390.478^{*}$ & 0.321 & -1.41 & $\mathrm{~b}$ \\
\hline La II & 6774.268 & 0.126 & -1.820 & b \\
\hline Ce II & 4515.849 & 1.058 & -0.240 & d \\
\hline Ce II & 4523.075 & 0.516 & -0.240 & $\mathrm{c}$ \\
\hline Ce II & 4562.359 & 0.478 & 0.210 & $\mathrm{~b}$ \\
\hline Ce II & 4628.239 & 1.366 & -0.430 & d \\
\hline Ce II & 4773.941 & 0.924 & -0.390 & $\mathrm{~b}$ \\
\hline Ce II & 5274.229 & 1.044 & -0.170 & $\mathrm{c}$ \\
\hline Ce II & 5472.279 & 1.247 & -0.100 & d \\
\hline Ce II & 5975.818 & 1.327 & -0.450 & $\mathrm{~b}$ \\
\hline Ce II & 6043.373 & 1.206 & -0.480 & $\mathrm{a}$ \\
\hline $\mathrm{Nd}$ II & 4645.760 & 0.559 & -0.760 & $\mathrm{~d}$ \\
\hline
\end{tabular}

Notes. The table lists: wavelength $\lambda$, excitation potential $\chi_{\text {exc }}$, adopted oscillator strengths $\log g f$, and source: (a) Merle et al. (2016), (b) GES line list v5 (Heiter et al. 2015), (c) adjusted to reproduce solar and/or Arcturus abundances, and (d) VALD. The effective log $g f$ is given for lines whose hyperfine structure was taken into account (identified with an asterisk after the wavelength).

standard error on the mean can be obtained with $k_{\alpha, N-1} \times s / \sqrt{N}$, where $k_{\alpha, N-1}$ is the percentile of the Student's $t$ distribution with $N-1$ degrees of freedom, such that the probability $P\left(-k_{\alpha, N-1}<\right.$ $\left.T<k_{\alpha, N-1}\right)$ is $1-\alpha$. For a two-sided test and $1-\alpha=0.70$ (i.e. approximately a $1 \sigma$ confidence interval $), k_{0.3,2}=1.386, k_{0.3,3}=$ $1.250, k_{0.3,4}=1.190, k_{0.3,7}=1.119, k_{0.3,8}=1.108$. This allows us to estimate a conservative random error for our abundance ratios of 0.1 dex.
Table B.3 facilitates the evaluation of the systematic errors on the final abundance ratios caused by the uncertainty on the stellar parameters. As expected, neutral species are more sensitive to the temperature than ionised species, while the tendency is opposite for gravity. Except for the notable case of $\mathrm{Zr} \mathrm{I}$, the impact of the model-atmosphere uncertainties is always below $0.1 \mathrm{dex}$, in absolute value, which we adopt as a conservative estimate of the systematic error on the abundances. 
M. Van der Swaelmen et al.: Orbital properties of barium and S stars, and red giants in open clusters
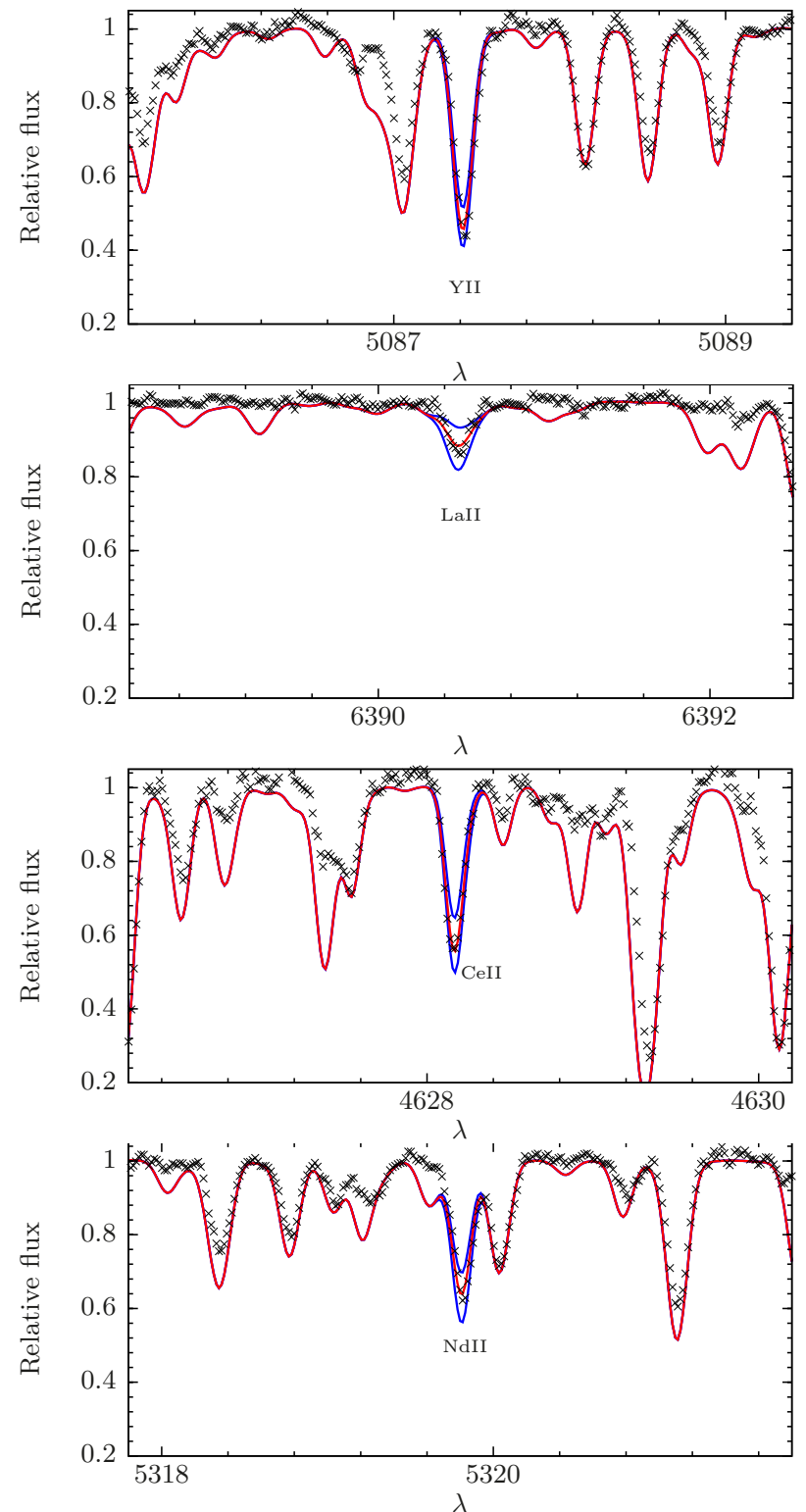

Fig. B.1. From top to bottom: fit of the Y II line at $508.7 \mathrm{~nm}$, La II line at $639.0 \mathrm{~nm}$, Ce II line at $462.8 \mathrm{~nm}$, and Nd II line at $531.9 \mathrm{~nm}$ for IC 4756.69 . Black crosses: observed spectrum; red solid line: best fit; blue solid lines: spectra computed around the best fit value $[\mathrm{X} / \mathrm{Fe}] \pm 0.3 \mathrm{dex}$. 
Table B.2. Line-by-line $[\mathrm{X} / \mathrm{Fe}]$ abundance ratios for $\mathrm{Y}, \mathrm{Zr}$, La, Ce, and $\mathrm{Nd}$ for open cluster stars falling in the post-mass-transfer region of the $(P-e)$ diagram.

\begin{tabular}{|c|c|c|c|c|c|c|c|c|}
\hline Ion & $\lambda$ & Sun & IC 4756139 & NGC 2099149 & NGC 2099966 & NGC 23354 & NGC 2420173 & NGC 2539209 \\
\hline$[\mathrm{Fe} / \mathrm{H}]$ & & 0.0 & -0.06 & +0.08 & +0.08 & -0.03 & -0.26 & +0.13 \\
\hline Y II & 4883.68 & -0.03 & +0.06 & +0.12 & +0.17 & +0.79 & +0.99 & -0.20 \\
\hline Y II & 5087.41 & +0.00 & -0.25 & -0.09 & -0.28 & +1.28 & +1.65 & -0.11 \\
\hline Y II & 5200.41 & +0.00 & - & +0.02 & - & +1.28 & +1.15 & -0.18 \\
\hline Y II & 5289.82 & +0.00 & - & - & - & +0.63 & - & - \\
\hline Y II & 5320.78 & - & +0.05 & -0.16 & -0.14 & - & +0.62 & -0.04 \\
\hline Y II & 5402.77 & +0.01 & -0.04 & - & -0.25 & -0.02 & +1.04 & -0.04 \\
\hline Y II & 5544.61 & +0.00 & +0.07 & -0.09 & +0.04 & -0.06 & +0.88 & +0.02 \\
\hline Y II & 5546.01 & +0.01 & +0.12 & +0.01 & -0.08 & -0.14 & +0.88 & - \\
\hline Y II & 5728.89 & +0.01 & +0.09 & +0.13 & +0.32 & +0.36 & +1.04 & +0.24 \\
\hline Y II & 6795.41 & -0.05 & +0.07 & +0.05 & +0.09 & +0.04 & +0.74 & +0.16 \\
\hline [Y II/Fe] & & $\begin{array}{l}-0.01 \\
\pm 0.02\end{array}$ & $\begin{array}{r}+0.02 \\
\pm 0.11\end{array}$ & $\begin{array}{r}+0.00 \\
\pm 0.10\end{array}$ & $\begin{array}{r}-0.02 \\
\pm 0.20\end{array}$ & $\begin{array}{r}+0.46 \\
\pm 0.53\end{array}$ & $\begin{array}{r}+1.00 \\
\pm 0.28\end{array}$ & $\begin{array}{r}-0.02 \\
\pm 0.15\end{array}$ \\
\hline Zr I & 4772.32 & +0.10 & - & - & -0.22 & - & - & -0.35 \\
\hline Zr I & 6127.48 & -0.04 & +0.43 & +0.01 & -0.16 & - & +0.72 & -0.30 \\
\hline Zr I & 6134.59 & +0.02 & +0.26 & - & - & - & +0.71 & - \\
\hline Zr I & 6143.25 & - & +0.32 & -0.07 & -0.21 & - & +0.72 & - \\
\hline [Zr I/Fe] & & $\begin{array}{r}+0.03 \\
\pm 0.06\end{array}$ & $\begin{array}{r}+0.34 \\
\pm 0.07\end{array}$ & $\begin{array}{r}-0.03 \\
\pm 0.04\end{array}$ & $\begin{array}{r}-0.20 \\
\pm 0.03\end{array}$ & - & $\begin{array}{r}+0.72 \\
\pm 0.00\end{array}$ & $\begin{array}{r}-\mathbf{0 . 3 2} \\
\pm 0.02\end{array}$ \\
\hline$[\mathrm{Zr} \mathbf{I I} / \mathbf{F e}]$ & 5112.27 & -0.01 & - & -0.18 & - & - & - & -0.10 \\
\hline La II & 4558.46 & -0.02 & - & - & - & - & - & - \\
\hline La II & 4574.86 & +0.00 & +0.19 & +0.31 & +0.18 & - & +0.47 & -0.20 \\
\hline La II & 4662.49 & +0.17 & +0.47 & - & +0.26 & - & - & +0.00 \\
\hline La II & 4748.73 & +0.11 & +0.20 & - & +0.13 & +0.20 & +0.71 & +0.07 \\
\hline La II & 4804.04 & - & +0.25 & +0.13 & +0.21 & - & +0.69 & +0.18 \\
\hline La II & 4920.98 & -0.08 & +0.47 & - & - & - & - & - \\
\hline La II & 5114.56 & - & +0.50 & +0.00 & +0.55 & - & +0.86 & +0.20 \\
\hline La II & 5290.82 & +0.02 & - & -0.15 & +0.21 & +0.22 & - & -0.02 \\
\hline La II & 5303.53 & -0.03 & +0.27 & -0.05 & +0.03 & +0.21 & +0.39 & +0.08 \\
\hline La II & 5797.57 & - & +0.37 & +0.06 & -0.07 & - & +0.62 & - \\
\hline La II & 5880.63 & - & +0.38 & +0.10 & +0.06 & +0.21 & - & - \\
\hline La II & 6390.50 & -0.04 & +0.37 & +0.02 & +0.21 & +0.02 & +0.58 & +0.11 \\
\hline La II & 6774.27 & & +0.35 & +0.29 & +0.39 & - & - & +0.17 \\
\hline [LaII/Fe] & & $\begin{array}{l}+\mathbf{0 . 0 4} \\
\pm \mathbf{0 . 0 7}\end{array}$ & $\begin{array}{r}+0.35 \\
\pm 0.10\end{array}$ & $\begin{array}{l}+0.08 \\
\pm 0.14\end{array}$ & $\begin{array}{r}+0.20 \\
\pm 0.16\end{array}$ & $\begin{array}{l}+0.17 \\
\pm 0.08\end{array}$ & $\begin{array}{r}+0.62 \\
\pm 0.15\end{array}$ & $\begin{array}{r}+\mathbf{0 . 0 7} \\
\pm \mathbf{0 . 1 2}\end{array}$ \\
\hline Ce II & 4515.85 & +0.13 & +0.00 & - & -0.01 & - & - & - \\
\hline Ce II & 4523.08 & +0.00 & +0.19 & -0.12 & -0.18 & - & +0.56 & - \\
\hline Ce II & 4562.36 & -0.11 & +0.02 & +0.03 & - & - & - & -0.29 \\
\hline Ce II & 4628.24 & - & - & - & - & - & - & \\
\hline Ce II & 4773.94 & - & +0.24 & +0.22 & - & +0.35 & - & +0.27 \\
\hline Ce II & 5274.23 & +0.01 & +0.31 & +0.37 & +0.24 & +0.33 & +0.75 & +0.10 \\
\hline Ce II & 5472.28 & - & +0.03 & -0.17 & -0.29 & -0.20 & - & -0.15 \\
\hline Ce II & 5975.82 & - & +0.42 & +0.16 & +0.17 & +0.12 & - & +0.10 \\
\hline Ce II & 6043.37 & -0.16 & +0.27 & +0.05 & +0.11 & - & +0.47 & +0.09 \\
\hline [CeII/Fe] & & $\begin{array}{r}-0.03 \\
\pm 0.10\end{array}$ & $\begin{array}{r}+0.18 \\
\pm 0.14\end{array}$ & $\begin{array}{r}+0.08 \\
\pm 0.18\end{array}$ & $\begin{array}{r}+0.01 \\
\pm 0.19\end{array}$ & $\begin{array}{r}+0.15 \\
\pm 0.22\end{array}$ & $\begin{array}{r}+0.59 \\
\pm 0.12\end{array}$ & $\begin{array}{r}+0.02 \\
\pm 0.18\end{array}$ \\
\hline [NdII/Fe] & 4645.76 & - & +0.36 & +0.06 & +0.08 & +0.63 & +1.51 & - \\
\hline
\end{tabular}

Notes. The cluster metallicities are adopted from the WEDBA database. 
M. Van der Swaelmen et al.: Orbital properties of barium and S stars, and red giants in open clusters

Table B.2. continued.

\begin{tabular}{|c|c|c|c|c|c|}
\hline Ion & $\lambda$ & NGC 2682143 & NGC 2682170 & NGC 2682244 & NGC 6940111 \\
\hline$[\mathrm{Fe} / \mathrm{H}]$ & & +0.00 & +0.00 & +0.00 & +0.01 \\
\hline Y II & 4883.68 & +0.01 & - & -0.31 & -0.05 \\
\hline Y II & 5087.41 & - & -0.59 & -0.34 & -0.18 \\
\hline Y II & 5200.41 & -0.31 & - & -0.44 & -0.23 \\
\hline Y II & 5289.82 & +0.09 & -0.03 & -0.03 & - \\
\hline Y II & 5320.78 & - & -0.23 & -0.07 & -0.05 \\
\hline Y II & 5402.77 & -0.13 & - & -0.19 & +0.07 \\
\hline Y II & 5544.61 & - & -0.02 & +0.06 & +0.17 \\
\hline Y II & 5546.01 & - & - & +0.11 & +0.10 \\
\hline Y II & 5728.89 & +0.44 & +0.17 & -0.03 & +0.28 \\
\hline Y II & 6795.41 & +0.26 & +0.05 & +0.06 & +0.17 \\
\hline [Y II/Fe] & & $\begin{array}{l}+0.06 \\
\pm 0.25\end{array}$ & $\begin{array}{l}-0.11 \\
\pm 0.25\end{array}$ & $\begin{array}{l}-0.12 \\
\pm 0.18\end{array}$ & $\begin{array}{c}+0.03 \\
\pm 0.16\end{array}$ \\
\hline $\begin{array}{r}\mathrm{Zr} \mathrm{I} \\
\mathrm{Zr} \mathrm{I} \\
\mathrm{Zr} \mathrm{I} \\
\mathrm{Zr} \mathrm{I} \\
{[\mathrm{Zr} \mathrm{I/Fe]}}\end{array}$ & $\begin{array}{l}4772.32 \\
6127.48 \\
6134.59 \\
6143.25\end{array}$ & $\begin{array}{r}+0.12 \\
+0.09 \\
+0.30 \\
- \\
+\mathbf{0 . 1 7} \\
\pm \mathbf{0 . 0 9}\end{array}$ & $\begin{array}{l}-0.36 \\
-0.09 \\
-0.22 \\
-0.36 \\
-\mathbf{0 . 2 6} \\
\pm \mathbf{0 . 1 1}\end{array}$ & $\begin{array}{l}+0.10 \\
+0.32 \\
+0.17 \\
+0.09 \\
+\mathbf{0 . 1 7} \\
\pm \mathbf{0 . 0 9}\end{array}$ & $\begin{array}{r}- \\
+0.49 \\
+0.37 \\
+0.32 \\
+\mathbf{0 . 3 9} \\
+\mathbf{0 . 0 7}\end{array}$ \\
\hline [Zr II/Fe] & 5112.27 & - & -0.23 & -0.28 & -0.10 \\
\hline La II & 4558.46 & - & - & - & -0.18 \\
\hline La II & 4574.86 & - & -0.24 & -0.19 & +0.11 \\
\hline La II & 4662.49 & - & -0.16 & -0.11 & +0.11 \\
\hline La II & 4748.73 & +0.03 & +0.02 & -0.17 & +0.15 \\
\hline La II & 4804.04 & - & -0.11 & -0.04 & +0.17 \\
\hline La II & 4920.98 & - & - & - & +0.18 \\
\hline La II & 5114.56 & - & -0.02 & -0.03 & +0.23 \\
\hline La II & 5290.82 & +0.42 & -0.07 & -0.16 & +0.18 \\
\hline La II & 5303.53 & +0.20 & -0.13 & -0.16 & - \\
\hline La II & 5797.57 & - & - & +0.02 & +0.20 \\
\hline La II & 5880.63 & - & - & - & +0.28 \\
\hline La II & 6390.50 & +0.28 & -0.04 & +0.07 & +0.30 \\
\hline La II & 6774.27 & - & -0.13 & - & +0.39 \\
\hline [LaII/Fe] & & $\begin{array}{l}+0.23 \\
\pm 0.14\end{array}$ & $\begin{array}{l}-\mathbf{0 . 1 0} \\
\pm \mathbf{0 . 0 7}\end{array}$ & $\begin{array}{r}-0.08 \\
\pm 0.08\end{array}$ & $\begin{array}{c}+0.18 \\
\pm 0.13\end{array}$ \\
\hline $\mathrm{Ce}$ II & 4515.85 & - & - & - & - \\
\hline Ce II & 4523.08 & -0.11 & - & -0.35 & -0.23 \\
\hline $\mathrm{Ce}$ II & 4562.36 & -0.26 & - & -0.44 & -0.09 \\
\hline $\mathrm{Ce}$ II & 4628.24 & - & - & - & - \\
\hline Ce II & 4773.94 & +0.37 & -0.13 & +0.24 & +0.31 \\
\hline Ce II & 5274.23 & +0.04 & -0.39 & -0.01 & +0.29 \\
\hline Ce II & 5472.28 & - & -0.46 & - & -0.13 \\
\hline Ce II & 5975.82 & - & - & +0.16 & +0.32 \\
\hline Ce II & 6043.37 & +0.18 & -0.17 & +0.05 & +0.24 \\
\hline [CeII/Fe] & & $\begin{array}{l}+0.04 \\
\pm 0.22\end{array}$ & $\begin{array}{r}-0.29 \\
\pm 0.14\end{array}$ & $\begin{array}{r}-0.06 \\
\pm 0.25\end{array}$ & $\begin{array}{c}+0.10 \\
\pm 0.22\end{array}$ \\
\hline [NdII/Fe] & 4645.76 & +0.22 & - & +0.06 & +0.21 \\
\hline
\end{tabular}


Table B.2. continued.

\begin{tabular}{|c|c|c|c|c|c|c|c|}
\hline Ion & $\lambda$ & IC 475669 & IC 475680 & NGC 24771044 & NGC 2682224 & NGC 4349203 & NGC 752208 \\
\hline$[\mathrm{Fe} / \mathrm{H}]$ & & -0.06 & -0.06 & +0.01 & +0.00 & -0.07 & -0.08 \\
\hline Y II & 4883.68 & -0.05 & -0.26 & +0.52 & -0.13 & +0.26 & -0.13 \\
\hline Y II & 4900.12 & - & - & +0.17 & -0.51 & -0.02 & -0.37 \\
\hline Y II & 5087.41 & -0.03 & - & +0.36 & - & +0.13 & -0.29 \\
\hline Y II & 5200.41 & - & - & +0.47 & - & +0.16 & - \\
\hline Y II & 5289.82 & +0.09 & +0.08 & +0.57 & -0.04 & +0.07 & +0.05 \\
\hline Y II & 5402.77 & +0.09 & +0.01 & +0.55 & +0.01 & +0.02 & +0.01 \\
\hline Y II & 5544.61 & +0.08 & -0.01 & +0.54 & +0.07 & +0.05 & +0.08 \\
\hline Y II & 5728.89 & +0.26 & +0.18 & +0.72 & +0.23 & +0.19 & +0.22 \\
\hline Y II & 6795.41 & +0.18 & +0.18 & +0.58 & +0.01 & +0.09 & +0.10 \\
\hline [Y II/Fe] & & $\begin{array}{r}+0.09 \\
\pm \mathbf{0 . 1 0}\end{array}$ & $\begin{array}{r}+0.03 \\
+0.15\end{array}$ & $\begin{array}{r}+0.54 \\
\pm \mathbf{0 . 1 0}\end{array}$ & $\begin{array}{r}+0.03 \\
\pm 0.11\end{array}$ & $\begin{array}{r}+0.12 \\
+0.07\end{array}$ & $\begin{array}{r}+0.01 \\
\pm \mathbf{0 . 1 5}\end{array}$ \\
\hline $\mathrm{Zr} \mathrm{I}$ & 4739.48 & - & - & -0.06 & -0.22 & -0.39 & -0.21 \\
\hline $\mathrm{Zr} \mathrm{I}$ & 4772.32 & -0.38 & -0.30 & +0.04 & -0.04 & -0.21 & -0.08 \\
\hline $\mathrm{Zr} \mathrm{I}$ & 5385.15 & - & - & +0.07 & -0.01 & - & -0.07 \\
\hline $\mathrm{Zr} \mathrm{I}$ & 5735.69 & +0.79 & +0.80 & +1.05 & +1.06 & +0.97 & +0.91 \\
\hline $\mathrm{Zr} \mathrm{I}$ & 6127.48 & -0.18 & -0.19 & +0.23 & - & -0.08 & - \\
\hline $\mathrm{Zr} \mathrm{I}$ & 6134.59 & -0.32 & -0.39 & +0.21 & +0.14 & -0.14 & +0.09 \\
\hline $\mathrm{Zr} \mathrm{I}$ & 6140.54 & - & - & +0.48 & - & - & +0.27 \\
\hline $\mathrm{Zr} \mathrm{I}$ & 6143.25 & - & - & +0.22 & +0.10 & - & +0.01 \\
\hline Zr I & 6445.75 & - & - & +0.33 & +0.20 & - & - \\
\hline $\mathrm{Zr} \mathrm{I}$ & 6990.87 & - & - & - & +0.35 & - & +0.83 \\
\hline$[\mathrm{Zr} \mathbf{I} / \mathbf{F e}]$ & & $\begin{array}{l}-0.02 \\
\pm 0.47\end{array}$ & $\begin{array}{r}-0.02 \\
\pm 0.48\end{array}$ & $\begin{array}{r}+0.29 \\
\pm 0.31\end{array}$ & $\begin{array}{r}+0.20 \\
\pm 0.36\end{array}$ & $\begin{array}{r}+0.03 \\
\pm 0.48\end{array}$ & $\begin{array}{c}+0.22 \\
\pm 0.40\end{array}$ \\
\hline $\mathrm{Zr}$ II & 5112.27 & +0.12 & +0.01 & +0.45 & -0.16 & +0.04 & -0.03 \\
\hline $\mathrm{Zr}$ II & 5350.09 & +0.35 & +0.21 & +0.75 & +0.16 & +0.37 & +0.22 \\
\hline $\mathrm{Zr}$ II & 5350.35 & +0.35 & +0.21 & +0.75 & +0.17 & +0.37 & +0.23 \\
\hline$[\mathrm{Zr} \mathbf{I I} / \mathbf{F e}]$ & & $\begin{array}{r}+0.27 \\
\pm \mathbf{0 . 1 1}\end{array}$ & $\begin{array}{r}+0.14 \\
\pm 0.09\end{array}$ & $\begin{array}{r}+0.65 \\
\pm 0.14\end{array}$ & $\begin{array}{r}+0.06 \\
\pm 0.15\end{array}$ & $\begin{array}{r}+0.26 \\
\pm 0.16\end{array}$ & $\begin{array}{r}+0.14 \\
\pm 0.12\end{array}$ \\
\hline La II & 4558.46 & +0.07 & - & +0.17 & - & -0.02 & +0.00 \\
\hline La II & 4574.86 & +0.10 & -0.08 & +0.47 & +0.16 & +0.23 & +0.27 \\
\hline La II & 4662.49 & +0.15 & -0.02 & +0.26 & - & +0.09 & +0.09 \\
\hline La II & 4748.73 & +0.10 & +0.01 & +0.20 & -0.11 & +0.01 & -0.00 \\
\hline La II & 4804.04 & +0.16 & +0.15 & +0.36 & +0.03 & +0.05 & +0.19 \\
\hline La II & 4920.98 & - & - & - & +0.10 & +0.02 & +0.16 \\
\hline La II & 5114.56 & +0.27 & +0.20 & +0.62 & +0.19 & +0.42 & +0.33 \\
\hline La II & 5290.82 & -0.05 & -0.16 & +0.21 & +0.01 & -0.04 & +0.07 \\
\hline La II & 5303.53 & +0.05 & +0.05 & +0.34 & +0.00 & +0.05 & +0.09 \\
\hline La II & 5880.63 & - & - & +0.45 & +0.01 & -0.05 & +0.02 \\
\hline La II & 5936.20 & - & - & +0.24 & +0.13 & - & +0.22 \\
\hline La II & 6172.70 & +0.20 & +0.19 & +0.54 & +0.40 & +0.21 & +0.26 \\
\hline La II & 6390.50 & +0.10 & +0.12 & +0.39 & +0.11 & +0.09 & +0.16 \\
\hline La II & 6774.27 & +0.15 & +0.18 & +0.46 & +0.07 & +0.23 & +0.20 \\
\hline [La II/Fe] & & +0.12 & +0.07 & +0.36 & +0.09 & +0.11 & +0.15 \\
\hline & & $\pm \mathbf{0 . 0 8}$ & $\pm \mathbf{0 . 1 2}$ & $\pm \mathbf{0 . 1 3}$ & $\pm \mathbf{0 . 1 2}$ & $\pm \mathbf{0 . 1 3}$ & $\pm \mathbf{0 . 1 0}$ \\
\hline
\end{tabular}


M. Van der Swaelmen et al.: Orbital properties of barium and S stars, and red giants in open clusters

Table B.2. continued.

\begin{tabular}{|c|c|c|c|c|c|c|c|}
\hline Ion & $\lambda$ & IC 475669 & IC 475680 & NGC 24771044 & NGC 2682224 & NGC 4349203 & NGC 752208 \\
\hline Ce II & 4515.85 & -0.23 & -0.36 & +0.07 & -0.22 & -0.21 & -0.18 \\
\hline Ce II & 4523.08 & -0.04 & -0.11 & +0.15 & -0.13 & -0.08 & -0.04 \\
\hline Ce II & 4628.16 & -0.14 & -0.27 & -0.02 & -0.33 & -0.10 & -0.21 \\
\hline Ce II & 4773.94 & +0.21 & +0.20 & +0.41 & +0.27 & +0.11 & +0.29 \\
\hline Ce II & 5187.46 & -0.14 & -0.14 & -0.03 & -0.24 & -0.13 & -0.13 \\
\hline Ce II & 5274.23 & +0.13 & +0.15 & +0.28 & -0.06 & +0.19 & +0.11 \\
\hline Ce II & 5330.57 & -0.06 & -0.03 & - & -0.14 & - & -0.06 \\
\hline Ce II & 5472.28 & -0.09 & -0.10 & +0.06 & -0.19 & -0.24 & -0.14 \\
\hline Ce II & 6043.37 & +0.09 & +0.13 & +0.34 & +0.05 & +0.03 & +0.11 \\
\hline Ce II & 6051.82 & - & - & +0.15 & -0.22 & - & -0.15 \\
\hline$[\mathrm{Ce}$ II/Fe] & & $-\mathbf{0 . 0 3}$ & -0.06 & +0.16 & -0.12 & -0.05 & -0.04 \\
\hline & & $\pm \mathbf{0 . 1 4}$ & $\pm \mathbf{0 . 1 8}$ & \pm 0.15 & \pm 0.16 & \pm 0.14 & \pm 0.15 \\
\hline [Nd II/Fe] & 4645.76 & +0.08 & +0.10 & +0.26 & +0.14 & +0.05 & +0.08 \\
\hline
\end{tabular}

Table B.3. Abundance uncertainties (in dex) for the star IC 4756.69, for uncertainties of $\Delta T_{\text {eff }}=150 \mathrm{~K}, \Delta \log g=0.2, \Delta[\mathrm{M} / \mathrm{H}]=0.1 \mathrm{dex}$, and $\Delta v_{\mathrm{t}}=0.2 \mathrm{~km} \mathrm{~s}^{-1}$.

\begin{tabular}{lllllllll}
\hline \hline Ion & $\Delta_{+}(T)$ & $\Delta_{-}(T)$ & $\Delta_{+}(\log g)$ & $\Delta_{-}(\log g)$ & $\Delta_{+}([\mathrm{M} / \mathrm{H}])$ & $\Delta_{-}([\mathrm{M} / \mathrm{H}])$ & $\Delta_{+}\left(v_{\mathrm{t}}\right)$ & $\Delta_{-}\left(v_{\mathrm{t}}\right)$ \\
\hline$[\mathrm{Y} \mathrm{II} / \mathrm{Fe}]$ & +0.02 & -0.02 & +0.08 & -0.09 & -0.07 & +0.08 & -0.09 & +0.10 \\
{$[\mathrm{Zr} \mathrm{I} / \mathrm{Fe}]$} & +0.22 & -0.26 & +0.00 & +0.00 & -0.12 & +0.10 & +0.00 & +0.00 \\
{$[\mathrm{Zr} \mathrm{II} / \mathrm{Fe}]$} & +0.00 & +0.01 & +0.10 & -0.10 & -0.09 & +0.09 & -0.02 & +0.03 \\
{$[\mathrm{La} \mathrm{II} / \mathrm{Fe}]$} & +0.04 & -0.05 & +0.09 & -0.10 & -0.09 & +0.08 & -0.01 & +0.01 \\
{$[\mathrm{Ce}$ II/Fe] } & +0.04 & -0.09 & +0.09 & -0.12 & -0.08 & +0.06 & -0.05 & +0.09 \\
{$[\mathrm{Nd}$ II/Fe] } & +0.06 & -0.04 & +0.10 & -0.08 & -0.06 & +0.09 & -0.06 & +0.08 \\
\hline
\end{tabular}

Notes. The value $\Delta_{ \pm} \zeta$ is defined as $[\mathrm{X} / \mathrm{Fe}](\zeta \pm \Delta \zeta)-[\mathrm{X} / \mathrm{Fe}]\left(\zeta_{\text {nominal }}\right)$, where $\mathrm{X}$ is the element under study and $\zeta$ is one of the four atmospheric parameters. 